\title{
Semiclassical Resonances Generated by a Closed Trajectory of Hyperbolic Type
}

\author{
C. Gérard ${ }^{1}$ and J. Sjöstrand ${ }^{2}$ \\ 1 Département de Mathématiques, Université de Paris Sud, F-91405 Orsay, France \\ 2 Department of Mathematics, University of Lund, Box 118, S-22100 Lund, Sweden
}

\begin{abstract}
We determine all the resonances in certain rectangular regions of the complex plane of the Schrödinger operator $-h^{2} \Delta+V$ when $h \rightarrow 0$, under the assumption that the set of trapped points of energy 0 for the classical flow form a closed trajectory and that the corresponding Poincaré map is hyperbolic.
\end{abstract}

\section{Introduction}

In this paper we consider a semiclassical differential operator $P$ on $\mathbb{R}^{n}$ with analytic coefficients, which satisfies all the general assumptions of [6, Sect. 8]. Let $p(x, \xi)$ be the principal symbol in the sense of $h$-pseudodifferential operators. [The most important special case is, of course, when $P=-h^{2} \Delta+V(x)$. Then $p=\xi^{2}+V(x)$.] We assume that

$$
p(x, \xi)=0 \Rightarrow d p \neq 0 .
$$

In the appendix of this paper, we give some generalities concerning the flow of $H_{p}=\sum p_{\xi_{j}}^{\prime} \partial_{x_{j}}-p_{x_{j}}^{\prime} \partial_{\xi_{j}}$ either in $p^{-1}\left(\left[-\varepsilon_{0}, \varepsilon_{0}\right]\right)$ or in $p^{-1}(0)$. For $\varrho \in T^{*} \mathbf{R}^{n}$, let ]$T_{-}(\varrho), T_{+}(\varrho)\left[\ni t \mapsto \exp t H_{p}(\varrho)\right.$ be the maximal classical trajectory. Here $T_{+}$and $-T_{-}$are lower semicontinuous functions of $\varrho$ with values in $\left.] 0,+\infty\right]$. We define the outgoing tail and the incoming tail by

$$
\tilde{\Gamma}_{ \pm}^{0}=\left\{\varrho \in p^{-1}(0) ; \exp t H_{p}(\varrho) \nrightarrow \infty, \text { as } t \rightarrow T_{\mp}(\varrho)\right\} .
$$

In the appendix we show among other things, that $K^{0}=\tilde{\Gamma}_{+}^{0} \cap \tilde{\Gamma}_{-}^{0}$ is a compact set. Our next assumption is then:

$$
\begin{gathered}
K^{0} \text { is (the image of) a simple closed trajectory } \\
\gamma^{0}:\left[0, T^{0}\right] \rightarrow p^{-1}(0) \quad\left[\text { satisfying } \gamma^{0}(0)=\gamma^{0}\left(T^{0}\right)\right] .
\end{gathered}
$$

Let $p^{0}$ be the corresponding linearized Poincaré map. It is a symplectic automorphism of the normal space of $\gamma^{0}$ in $p^{-1}(0)$ at the point $\gamma^{0}(0)$, defined as the differential of the smooth map $H^{0} \rightarrow H^{0}$, obtained by following the flow of $H_{p}$ once around $\gamma^{0}$. Here $H^{0} \subset p^{-1}(0)$ is some smooth hypersurface intersecting $\gamma^{0}$ 
transversally at $\gamma^{0}(0)$. If $\theta$ is an eigenvalue of $p^{0}$, then $1 / \theta, \bar{\theta}, 1 / \bar{\theta}$ are also eigenvalues of $p^{0}$ with the same multiplicity. We assume that our Poincaré map is hyperbolic:

$$
p^{0} \text { has no eigenvalues of modulus } 1 \text {. }
$$

In particular, $\operatorname{det}\left(p^{0}-I\right) \neq 0$, so by the implicit function theorem, there is an $\varepsilon_{0}>0$ and an analytic family of closed trajectories:

$$
\gamma^{\varepsilon}:\left[0, T^{\varepsilon}\right] \rightarrow p^{-1}(\varepsilon), \quad \varepsilon \in\left[-\varepsilon_{0}, \varepsilon_{0}\right],
$$

so that the corresponding Poincare maps are all hyperbolic.

Going back to our general discussion, we define $\widetilde{\Gamma}_{ \pm}^{\varepsilon}, K^{\varepsilon}=\widetilde{\Gamma}_{+}^{\varepsilon} \cap \widetilde{\Gamma}_{-}^{\varepsilon}$ in $p^{-1}(\varepsilon)$, for $\varepsilon \in\left[-\varepsilon_{0}, \varepsilon_{0}\right]$ just as in the case $\varepsilon=0$. It is then easy to see, at least after reading the appendix, that $K^{\delta} \rightarrow K^{\varepsilon}$ as $\delta \rightarrow \varepsilon$, in the sense that $K^{\delta}$ is contained in any given neighbourhood of $K^{\varepsilon}$, when $\delta$ is sufficiently close to $\varepsilon$. [Here we do not use the assumptions (0.3), (0.4).] If $G$ is any escape function (defined in [6]), then we have the same result concerning the families $\widetilde{\Gamma}_{ \pm}^{\varepsilon} \cap\{ \pm G \leqq T\}$, for every fixed $T$. On the other hand, it is easy to see that when $(0.3),(0.4)$ are satisfied, then there is a neighbourhood $U$ of $\bar{\gamma}=\bigcup_{-\varepsilon_{0} \leqq \varepsilon \leqq \varepsilon_{0}} \gamma^{\varepsilon}$ in $p^{-1}\left(\left[-\varepsilon_{0}, \varepsilon_{0}\right]\right)$, such that if $\varrho \in U$ and $\exp t H_{p}(\varrho) \in U$ for all $t \in \mathbf{R}$, then $\varrho \in \bar{\gamma}$. Then after decreasing $\varepsilon_{0}$ if necessary, we conclude that

$$
K^{\varepsilon}=\gamma^{\varepsilon}, \quad-\varepsilon_{0} \leqq \varepsilon \leqq \varepsilon_{0} .
$$

(Whenever it is convenient to do so, we shall identify $\gamma^{\varepsilon}$ with its image.)

It is easy to prove the following facts: (See [1] for $(0.8),(0.9)$ in the $C^{\infty}$-case. The proof of the appendix in [11] can be adapted to the present analytic case.)

$\bar{\gamma}$ is an analytic 2-dimensional symplectic manifold.

$J_{ \pm}=\bigcup \tilde{\Gamma}_{ \pm}^{\varepsilon} \quad$ are analytic involutive manifolds of dimension $n+1$, intersecting transversally along $\bar{\gamma}$.

$$
\Lambda_{ \pm}^{\varepsilon}=\tilde{\Gamma}_{ \pm}^{\varepsilon}=J_{ \pm} \cap p^{-1}(\varepsilon) \text { are Lagrangian analytic manifolds, }
$$
intersecting transversally in $p^{-1}(\varepsilon)$ along $\gamma^{\varepsilon}$.

At a point $x(\varepsilon)$ of $\gamma^{\varepsilon}$, we can write $T \Lambda_{ \pm}^{\varepsilon}=\mathbf{R} \dot{\gamma}_{\varepsilon} \oplus T \Gamma_{ \pm}^{\varepsilon}$, where $\Gamma_{ \pm}^{\varepsilon}$ are the bicharacteristic leaves of $J_{ \pm}$(of dimension $n-1$ ). $T \Gamma_{+}^{\varepsilon} \oplus T \Gamma_{-}^{\varepsilon}$ is then the spectral decomposition of $p^{\varepsilon}$ into spaces corresponding to eigenvalues of modulus $>1$ and $<1$, respectively. Let $D^{\varepsilon}=\left.p^{\varepsilon}\right|_{T \Gamma_{+}^{\varepsilon}}: T \Gamma_{+}^{\varepsilon} \rightarrow T \Gamma_{+}^{\varepsilon}$.

Let $D_{*}^{\varepsilon}: \mathscr{P}^{N-1}\left(T \Gamma_{+}^{\varepsilon}\right) \rightarrow \mathscr{P}^{N-1}\left(T \Gamma_{+}^{\varepsilon}\right)$ be defined by $\left(D_{*}^{\varepsilon} u\right)(t)=u\left(\left(D^{\varepsilon}\right)^{-1}(t)\right)$. Here $\mathscr{P}^{N-1}$ denotes the space of complex polynomials of degree $N-1$. If we identify $T \Gamma_{+}^{\varepsilon}$ suitably with $\mathbf{R}^{n-1}$, it is easy to see that $D^{\varepsilon}$ and $D_{*}^{\varepsilon}$ depend analytically on $\varepsilon$. The eigenvalues of $D^{\varepsilon}$ are, of course, the eigenvalues $\theta_{1}(\varepsilon), \ldots, \theta_{n-1}(\varepsilon)$ of $p$ of modulus $>1$. The eigenvalues of $D_{*}^{\varepsilon}$ are then the numbers $\theta(\varepsilon)^{-\alpha}=\theta_{1}(\varepsilon)^{-\alpha_{1}} \cdot \ldots \cdot \theta_{n-1}(\varepsilon)^{-\alpha_{n-1}}$, with $|\alpha|=\alpha_{1}+\ldots+\alpha_{n-1} \leqq N-1$. Here the algebraic multiplicity of each eigenvalue is the number of $\alpha$ 's of length $\leqq N-1$, which give rise to this value.

In the next section we shall define a certain analytic function $\varrho(\varepsilon)$ which satisfies

$$
|\varrho(\varepsilon)|=\left|\theta_{1}(\varepsilon) \cdot \ldots \cdot \theta_{n-1}(\varepsilon)\right|^{-1 / 2} .
$$


Let us also introduce the action

$$
C(\varepsilon)=\int_{\gamma^{\varepsilon}} \xi d x .
$$

As we shall see in the next section, we have

$$
C^{\prime}(\varepsilon)=T(\varepsilon) \text {. }
$$

For a complex value of $E$, we have

$$
\operatorname{det}\left(\varrho(E) D_{*}^{E}-e^{-i C(E) / h}\right)=0
$$

if and only if, for some $\alpha \in \mathbf{N}^{n-1}$ of length $\leqq N-1$, and for some $k \in \mathbf{Z}$, we have

$$
C(E)=2 \pi k h+i h \log \varrho(E)-i h \sum \alpha_{j} \log \theta_{j}(E) .
$$

From now on we restrict the attention to a rectangle $\left[-\varepsilon_{0}, \varepsilon_{0}\right]-i\left[0, C_{0} h\right]$, where $\varepsilon_{0}>0$ is sufficiently small, but independent of $h$, and $C_{0}$ may be arbitrarily large. Let $\Omega(h)$ be a subdomain such that

$$
\sup _{E \in \Omega(h)}\left|E-E_{1}\right| \rightarrow 0, \quad h \rightarrow 0,
$$

for some function $E_{1}(h)$ with real values. We assume moreover that

$$
\operatorname{det}\left(\varrho\left(E_{1}\right) D_{*}^{E_{1}}-e^{-i C(E) / h}\right) \geqq \text { const }>0,
$$

for all $E \in \partial \Omega(h)$. Then it is easy to see that there is a bijection $\kappa$ between the set of solutions to $(0.13)$ in $\Omega(h)$ counted with their multiplicity, and the set of solutions in $\Omega(h)$ to

$$
\operatorname{det}\left(\varrho\left(E_{1}\right) D_{*}^{E_{1}}-e^{-i C(E) / h}\right)=0,
$$

counted with their multiplicity, such that $\kappa(E)-E=o(h)$, uniformly with respect to $E$. Now (0.14) is equivalent to

$$
C(E)=2 \pi k h+i h \log \varrho\left(E_{1}\right)-i h \sum \alpha_{j} \log \theta_{j}\left(E_{1}\right),
$$

where the only implicit part is given by the inversion of the map $C$. This is no problem however, in view of (0.12). A simple consequence of this small discussion is that there exists a number $N_{0}$, such that no disc of radius $h$ can contain more than $N_{0}$ solutions (counted with multiplicity) of $(0.13)$.

Let $\Gamma^{0}(h)$ be the set of solutions of $(0.13)$ in $\left[-\varepsilon_{0}, \varepsilon_{0}\right]-i\left[0, C_{0} h\right]$, counted with their multiplicity. Then we have:

Theorem 1. For every $C_{0}>0$, if $N$ in the definition of $D_{*}^{E}$ is sufficiently large, we have: If $h$ is sufficiently small, there is an injective map $b(h)$ from $\Gamma^{0}(h)$ into the set of resonances of $P$ (counted with their multiplicity), such that $b(h)(\mu)-\mu=o(h)$, uniformly as $h \rightarrow 0$, and such that all resonances in $\left[-\varepsilon_{0}+\alpha h, \varepsilon_{0}-\alpha h\right]-i\left[0, C_{1} h\right]$ are in the image of $b(h)$. Here $\alpha>0$ and $0<C_{1}<C_{0}$ are fixed arbitrary constants.

There is also a more refined version of this result, which determines the resonances of $P$ (in the given rectangular region) not only modulo $o(h)$, but modulo $O\left(h^{\infty}\right)$. It is a little longer to formulate: 
Theorem 2. Fix $C_{0}>0$. Then there is a number $N \in \mathbf{N}$ and a matrix $F_{-+}^{\infty}(E, z, h): \mathscr{P}^{N-1} \rightarrow \mathscr{P}^{N-1}$ with the following properties:

$F_{-+}^{\infty}$ is holomorphic in $(E, z)$ in an open h-independent set

in $\mathbf{C}^{2}$ which contains

$$
\left(\left[-2 \varepsilon_{0}, 2 \varepsilon_{0}\right]+i\left[-2 \varepsilon_{0}, 2 \varepsilon_{0}\right]\right) \times\left\{z \in \mathbf{C} ; r_{0} \leqq|z| \leqq 1\right\} .
$$

Here $r_{0}$ is so small that $F_{-+}^{\infty}\left(E, e^{-i C(E) / h}, h\right)$ is well-defined for $E \in\left[-\varepsilon_{0}, \varepsilon_{0}\right]$ $-i\left[0, C_{0} h\right]$.

$$
\begin{gathered}
F_{-+}^{\infty} \text { is a symbol; } \quad F_{-+}^{\infty} \sim \sum_{0}^{\infty} A_{j}(E, z) h^{j / 2}, \\
A_{0}(E, z)=z-\varrho(E) D_{*}^{E} .
\end{gathered}
$$

Let $\Gamma^{\infty}(h)$ be the set of $E$ in the basic rectangle such that $\operatorname{det} F_{-+}^{\infty}\left(E, e^{-i C(E) / h}, h\right)=0$. Then there is an injective map $b(h)$ from $\Gamma^{\infty}(h)$ into the set of resonances of $P$ (where the elements of both sets are counted with their natural multiplicity), such that $b(h)(\mu)-\mu=O\left(h^{M}\right)$ uniformly for each $M$, and such that for every $M$, the image of $b(h)$ contains all resonances in the slightly smaller rectangle $\left[-\varepsilon_{0}+h^{M}, \varepsilon_{0}-h^{M}\right]$ $-i\left[0, C_{0} h-h^{M}\right]$, when $h$ is small enough. (See also Remark 5.2.)

We became interested in this problem through a paper of Ikawa [7], dealing with resonances for the Dirichlet problem for the Laplacian in the exterior of two strictly convex obstacles. Roughly, one could say that Ikawa obtained the first string of resonances (closest to the real axis) corresponding to our Theorem 1, given by (0.13) with $\alpha=0$. Later Ikawa [8] and C. Gérard improved this result. Ikawa got the complete asymptotics of the first string of resonances, while Gérard [4] got the complete asymptotics for all the strings, which is the complete analogue of our Theorem 2. Guillopé has also got analogous results in an explicitly computable situation [5].

The method we use here has many similarities with the method of Gerard. In particular, we make use of a certain associated Grushin problem. Technically, there are also many differences, and we use here some techniques from the study of operators with multiple characteristics as in Boutet de Monvel-Grigis-Helffer [2], or Sjöstrand [12] (containing also references to the work of Grushin), combined with more recent work on microlocal analytic singularities $[11,13]$.

This result can be compared with earlier results on quasimodes associated with closed elliptic trajectories (Ralston [15], Voros [18]) or with invariant Lagrangian manifolds (Colin de Verdière [16], Candelpergher-Nosmas [17]), which provide sequences of real eigenvalues for self adjoint operators. As was remarked long ago by Duistermaat [19] the construction of quasimodes associated with the stable or unstable manifolds of a closed hyperbolic trajectory does not work because there is no smooth $H_{p}$ invariant density on them. On the other hand, the construction of quasimodes living just on the closed trajectory give complex eigenvalues. The result we obtain shows that these problems disappear in the framework of resonances and that the quasimodes give all the resonances in a box $\left[-\varepsilon_{0}+a h, \varepsilon_{0}-a h\right]+i\left[0,-C_{0} h\right]$. 
We give some examples. The basic mathematical example is $P=-h^{2} \Delta+V(x)$ on $\mathbf{R}^{n}$ with

$$
V(x)=x_{1}{ }^{2}-\sum_{2}^{n} x_{i}^{2}
$$

near an energy level $E>0$. The closed hyperbolic trajectory is:

$$
\left(x_{1}, \xi_{1}\right)=\exp (-2 i t)\left(x_{1}(0), \xi_{1}(0)\right) ; \quad x_{2}=\ldots=x_{n}=0 ; \quad \xi_{2}=\ldots=\xi_{n}=0 .
$$

The physical analogue of this example is $P=-h^{2} \Delta+V(x)$ on $\mathbf{R}^{n}$ with $V(x)=|r|^{-1}+a x_{1}$ (repulsive Coulomb potential perturbed by Stark effect), near an energy level $E>2 \sqrt{a}$.

Finally, let us mention that these kind of resonances have already been observed by Pollak (see [20]).

The plan of the paper is the following: In Sect. 1 we study a certain Poincaré operator, acting on symbols, and we define the quantity $\varrho(E)$. In Sect. 2 we introduce a certain transformation, which is well-defined on outgoing functions (but not on general $L^{2}$-functions). We then get a simple form for the operator near $\bar{\gamma}$. In Sect. 3 we give estimates for a certain Grushin problem associated to a pseudodifferential operator in 1 dimension. At the end of Sect. 4 we combine the results of Sects. 3 and 4, in order to obtain a well-posed Grushin problem for the original operator. In Sect. 5, we derive some additional asymptotic information about the solution to this Grushin problem and we prove Theorem 2, which implies Theorem $1 .^{1}$

\section{Computation of a Principal Symbol}

Some parts of this discussion could also be carried out in a more standard way, by introducing the subprincipal symbol of the operator and interpreting certain leading symbols as half-densities.

We may choose real symplectic coordinates $(x, \xi)$ centered at $\gamma^{0}(0)=(0,0)$ such that $p=\xi_{1}, J_{+}: \xi^{\prime \prime}=0, J_{-}: x^{\prime \prime}=0$. Here we use the notation $x=\left(x_{1}, x^{\prime \prime}\right), \xi=\left(\xi_{1}, \xi^{\prime \prime}\right)$. The symplectic map $\kappa=\exp T_{0} H_{p}$, restricted to $\bar{\gamma}: x^{\prime \prime}=\xi^{\prime \prime}=0$, then maps $\left(x_{1}, \xi_{1}\right)$ to $\left(x_{1}+f\left(\xi_{1}\right), \xi_{1}\right)$. We also know that the bicharacteristic leaves of $J_{ \pm}$are mapped onto bicharacteristic leaves, so if $D$ is the differential of $\kappa$ at $(0,0)$, then $D$ maps: $\delta_{x_{1}} \mapsto \delta_{x_{1}}, \delta_{\xi_{1}} \mapsto f^{\prime}(0) \delta_{x_{1}}+\delta_{\xi_{1}}, \delta_{x^{\prime \prime}} \mapsto A \delta_{x^{\prime \prime}}, \delta_{\xi^{\prime \prime}} \mapsto{ }^{t} A^{-1} \delta_{\xi^{\prime \prime}}$. Here $\left(\begin{array}{cc}A & 0 \\ 0 & { }^{t} A^{-1}\end{array}\right)$ is the linearized Poincare map $p^{0}$. If we write the coordinates in the order $\left(x_{1}, \xi_{1}, x^{\prime \prime}, \xi^{\prime \prime}\right)$, it follows that $D$ is given by the matrix

$$
\left(\begin{array}{cccc}
1 & f^{\prime}(0) & & \\
0 & 1 & & 0 \\
& 0 & A & 0 \\
0 & 0 & { }^{t} A^{-1}
\end{array}\right) .
$$

\footnotetext{
${ }^{1}$ We would like to thank the referee who has helped us to eliminate several misprints and make several statements more precise
} 
Consider the strictly positive Lagrangian plane $\Lambda_{\delta}: \eta=i \delta y$, given by the phase $i \delta y^{2} / 2$. (See Melin-Sjöstrand [9] and Sjöstrand [13] for terminology.)

Let $\tilde{\Lambda}_{\delta}$ be the image under $D$. Then by (1.1) we get the equations for $\tilde{\Lambda}_{\delta}$ :

$$
\xi^{\prime \prime}=i^{t} A^{-1} A^{-1} x^{\prime \prime}, \quad \xi_{1}=i \delta\left(1+i \delta f^{\prime}\right)^{-1} x_{1}=\left(i \delta+O\left(\delta^{2}\right)\right) x_{1} .
$$

We now introduce an FBI-transform

$$
T u(x, h)=\int e^{i \phi(x, y) / h} t(x, y, h) u(y) d y,
$$

as in $\left[13\right.$, Sect. 7] (with $\lambda=1 / h$ ). We could for instance take $\phi(x, y)=i(x-y)^{2} / 2$. Let $\kappa_{T}:\left(y,-\phi_{y}^{\prime}\right) \mapsto\left(x, \phi_{x}^{\prime}\right)$ be the corresponding canonical transformation. Then we know that $\kappa_{T}\left(\mathbf{R}^{2 n}\right)=\Lambda_{\Phi}: \xi=(2 / i) \partial \Phi / \partial x$, where $\Phi$ is a strictly plurisubharmonic function. [If $\phi=i(x-y)^{2} / 2$, then $\Phi=(\operatorname{Im} x)^{2} / 2$.] We denote $\kappa_{T} \gamma^{0}$ simply by $\gamma^{0}$. Then $\pi_{x}\left(\gamma^{0}\right)$ is a simple closed loop in $\mathbf{C}_{x}^{n}$. For short we also write $\Lambda_{ \pm}^{0}$ instead of $\kappa_{T} \Lambda_{ \pm}^{0}$. We then know that the complexifications: $\left(\Lambda_{ \pm}^{0}\right)^{\mathbf{C}}$ near $\gamma^{0}$ are given by:

$$
\xi=\frac{\partial}{\partial x} \phi_{ \pm}^{0}
$$

Here $\phi_{ \pm}^{0}$ are holomorphic with $-\operatorname{Im} \phi_{ \pm}^{0} \leqq \Phi$, and more precisely, $\Phi+\operatorname{Im} \phi_{ \pm}^{0}$ $\sim \operatorname{dist}\left(\cdot, L_{ \pm}^{0}\right)^{2}$, where $L_{ \pm}^{0}=\pi_{x}\left(\Lambda_{ \pm}^{0}\right)$. This follows from the fact that $\kappa_{T}\left(\left(\Lambda_{ \pm}^{0}\right)^{\mathbf{c}}\right)$ intersects $\Lambda_{\Phi}$ along $\kappa_{T}\left(\Lambda_{ \pm}^{\overline{0}}\right)$, and from wellknown inequalities between harmonic and strictly subharmonic functions. If $p$ also denotes the transformed principal symbol of $P$, then we have the eiconal equation:

$$
p\left(x,\left(\phi_{ \pm}^{0}\right)^{\prime}\right)=0 \text {. }
$$

We have to be a little careful, however. While $\operatorname{Im} \phi_{ \pm}^{0}$ are single valued (equal to $-\Phi$ on $L_{ \pm}^{0}$ ), there is no reason why $\operatorname{Re} \phi_{ \pm}^{0}$ should be so. In the above discussion we first notice that everything is uniformly valid if we replace the superscript " 0 " by " $\varepsilon$ ", for $\varepsilon$ in some complex neighborhood of 0 . We then have to replace (1.5) by the more general equation:

$$
p\left(x,\left(\phi_{ \pm}^{\varepsilon}\right)_{x}^{\prime}\right)=\varepsilon .
$$

If we lift ourselves to the universal covering space of a neighborhood of $\pi_{x}\left(\gamma^{0}\right)$, then we can introduce the map $\mathscr{T}$, which consists in "moving a point $x$ one loop around $\pi_{x}\left(\gamma^{0}\right)$ back to itself." For our multivalued function $\phi_{+}^{\varepsilon}$, we then have:

$$
\phi_{+}^{\varepsilon}(\mathscr{T} x)=\phi_{+}^{\varepsilon}(x)+C(\varepsilon),
$$

where

$$
C(\varepsilon)=\int_{\gamma^{\varepsilon}} \xi d x
$$

It is easy to see that the integral in (1.8) takes the same value for any globally defined symplectic coordinates on $\mathbf{R}^{2 n}$, so going back to the standard symplectic coordinates on $\mathbf{R}^{2 n}$, we see that $C(\varepsilon)$ is real for real $\varepsilon$.

Differentiating (1.6) with respect to $\varepsilon$, we get

$$
\sum_{1}^{n} \frac{\partial p}{\partial \xi_{j}} \frac{\partial}{\partial x_{j}}\left(\frac{\partial \phi_{+}^{\varepsilon}}{\partial \varepsilon}\right)=1,
$$


and if we restrict this to $\pi_{x}\left(\gamma^{\varepsilon}\right)$, we get the ordinary differential equation:

$$
\frac{d}{d t}\left(\frac{\partial \phi^{\varepsilon}}{\partial \varepsilon}\right)=1
$$

Here $t$ is the natural parametrization of $\gamma^{\varepsilon}$. If $T(\varepsilon)$ is the period of $\gamma^{\varepsilon}$, we get, after comparing the integrated version of (1.10) with the differentiated version of (1.7),

$$
C^{\prime}(\varepsilon)=T(\varepsilon) \text {. }
$$

We let $P$ also denote the pseudodifferential operator, obtained by conjugation with $T$. The principal symbol of $P$ is then the transformed principal symbol $p$. We now consider multivalued WKB-solutions defined near $\pi_{x}\left(\gamma^{0}\right)$ of the form $a^{\varepsilon}(x, h) \exp \left(i \phi_{+}^{\varepsilon}(x) / h\right)$ of

$$
(P-\varepsilon)\left(a e^{i \phi^{\varepsilon} / h}\right) \sim 0 .
$$

Here $a$ is a classical symbol of order 0 with holomorphic coefficients;

$$
a^{\varepsilon}(x, h) \sim \sum_{0}^{\infty} a_{j}(x) h^{j} .
$$

If $H$ is a complex hypersurface intersecting $\gamma^{0}$ transversally at the point $x_{0}$, then we get a unique solution to (1.12) (in the covering space) if we prescribe $\left.a\right|_{H}$. The leading symbol $a_{0}^{\varepsilon}$ solves the transport equation

$$
\mathscr{L} a_{0}^{\varepsilon}=\left(\sum_{1}^{n} \frac{\partial p}{\partial \xi_{j}} \partial_{x_{j}}+i p_{-1}\left(x, \phi_{+}^{\varepsilon \prime}\right)+\frac{1}{2} \sum \sum \frac{\partial^{2} p}{\partial \xi_{j} \partial \xi_{k}} \partial_{x_{j}} \partial_{x_{k}} \phi_{+}^{\varepsilon}\right) a_{0}^{\varepsilon}=0 .
$$

Restricting this to $\pi_{x}\left(\gamma^{\varepsilon}\right)$, we get an ordinary differential equation, and it follows that

$$
a_{0}^{\varepsilon}(\mathscr{T} x)=\varrho(\varepsilon) a_{0}^{\varepsilon}(x), \quad x \in \pi_{x}\left(\gamma^{\varepsilon}\right),
$$

where $\varrho(\varepsilon)$ is an analytic non-vanishing function.

It is easy to see that $\varrho(\varepsilon)$ is independent of $a_{0}$ and of the choice of FBI-transform. We could even define $\varrho(\varepsilon)$ by the natural WKB-procedure before FBItransformation, but we would then need Maslov theory because of the appearance of caustics.

Let $\psi_{\delta}=\phi_{+}^{0}+O\left(\delta\left|x-x_{0}\right|^{2}\right)$ be the phase associated to $\kappa_{T}\left(\Lambda_{\delta}\right)=\tilde{\Lambda}_{\delta}$. We can then construct a WKB-solution to

$$
\left(D_{t}+P\right)\left(b(t, x, h) e^{i \psi(\delta, t, x) / h}\right) \sim 0,
$$

with initial condition $\left.\psi\right|_{t=0}=\psi_{\delta}$. Here $\psi$ and $b$ are determined by the usual eiconal and transport equations. We have

$$
\psi(\delta, t, x)=\phi_{+}^{0}(x)+O\left(\delta\left|x-\pi_{x} \gamma(t)\right|^{2}\right),
$$

so if we write down the transport equation for the leading part of $b$ along the curve $t \mapsto\left(t, \pi_{x} \gamma(t)\right)$, we get

$$
\left(\partial_{t}+(\mathscr{L}+O(\delta)) b_{0}=0\right.
$$


Here $O(\delta)$ indicates a multiplicative term. We conclude that

$$
b_{0}\left(T_{0}, x_{0}\right)=(\varrho(0)+O(\delta)) b_{0}\left(0, x_{0}\right) .
$$

Let $u=a \exp \left(i \phi_{+}^{0} / h\right), v_{t}=b \exp (i \psi / h)$. From the self-adjoint character of $P$, it follows that

$$
\left(v_{0} \mid u\right)=\left(v_{T_{0}} \mid \mathscr{T}^{*} u\right)+O_{\delta}\left(h^{\infty}\right),
$$

where $\left(\mathscr{T}^{*} u\right)(x)=u(\mathscr{T} x)$, and where the scalar product on the FBI-transform side is the one naturally induced from the standard $L^{2}$ scalar product on $\mathbf{R}^{n}$, which makes $P$ self adjoint.

Now let us represent the relation (1.20) in the special real symplectic coordinates considered in the beginning of this section. When $u_{1}, u_{2}$ are suitable oscillatory functions, we can define the induced scalar product from $L^{2}\left(\mathbf{R}^{n}\right)$ [modulo $\left.O\left(h^{\infty}\right)\right]$ by:

$$
\left(\left(u_{1} \mid u_{2}\right)\right)=\left(A\left(x, \widetilde{D}_{x}, h\right) u_{1} \mid u_{2}\right)_{L^{2}},
$$

where $A$ is an elliptic formally self-adjoint pseudodifferential operator of order 0 . In this representation we have $u=a(x, h)$ and $\mathscr{T} * u$ $=e^{i C(0) / h} \varrho(0)\left(a(x, h)+O(h)+O\left(\left|x-x_{0}\right|\right)\right)$. We may take $v_{0}=e^{-\delta x^{2} / 2 h}$. Then $v_{T_{0}}=e^{i C(0) / h} b(\delta, x, h) \exp \left(-f_{\delta}(x) / 2 h\right)$, where $f_{\delta}(x)=\left(A^{-1} x^{\prime}\right)^{2}+(1+O(\delta)) x_{n}^{2}+O\left(|x|^{3}\right)$, and $b_{0}(\delta, 0)=\varrho(0)+O(\delta)$. Inserting all this into (1.20) [or rather its analogue for the scalar product (1.21)] and identifying the leading terms in $h$, we get

$$
\delta^{-n / 2} \overline{a_{0}(0)}=(1+O(\delta)) \delta^{-n / 2}|\varrho(0)|^{2} \overline{a_{0}(0)} \operatorname{det} A .
$$

Letting $\delta$ tend to 0 , we obtain,

$$
|\varrho(0)|^{2}=1 /|\operatorname{det} A|=1 /\left|\theta_{1}(0) \cdot \ldots \cdot \theta_{n-1}(0)\right| .
$$

This relation extends, of course, to the case when 0 is replaced by $\varepsilon$, and we get

Proposition 1.1. We have

$$
\left.\varrho \varrho(\varepsilon)|=| \prod_{1}^{n-1} \theta_{j}(\varepsilon)\right|^{-1 / 2} .
$$

Introducing the subprincipal symbol of $P$, one could also get a formula for $\arg \varrho(\varepsilon)$.

\section{An Additional Canonical Transformation}

In this section we construct new global symplectic coordinates near $\gamma^{0}$, where $J_{+}^{\mathbf{c}}$ and $J_{-}^{\mathbf{C}}$ will be given, respectively by $\xi_{2}=\ldots=\xi_{n}=0$ and $x_{2}=\ldots=x_{n}=0$, and $\left(\gamma^{0}\right)^{\mathbf{C}}$ will be just $S^{1} \times i \mathbf{R}$.

We then quantify this transformation by a Fourier integral operator in the complex domain (see [13]), which acts only on suitable outgoing functions. (The discussion of the geometry is invariant under $\kappa_{T}$ and we can identify $\mathbf{R}^{n}$ with $\Lambda_{\Phi}$.)

We start by choosing real global analytic coordinates $(t, \tau)$ on $\bar{\gamma}$ so that $\gamma^{0}$ is given by $\tau=0$, so that $\tau$ is single valued and so that $t$ is multivalued with $\mathscr{T} t=t+2 \pi$. This is easy, if we first choose $t$ and then solve $-H_{t}(\tau)=1,\left.\tau\right|_{\gamma^{0}}=0$. We 
introduce $q_{1}=t+i f(t, \tau)$, where $f$ is single valued and vanishes for $\tau=0$. We want $i^{-1}\left\{q_{1}, \bar{q}_{1}\right\}<0$, where the Poisson bracket is defined from the symplectic form on $\bar{\gamma}$. On $\gamma^{0}: \tau=0$, we get $i^{-1}\left\{q_{1}, \bar{q}_{1}\right\}=2 \partial_{\tau} f$, so we can take $f=-a \tau$, where $a>0$. Put $p_{1}=\tau$.

Having determined $q_{1}$ on $\bar{\gamma}$, we extend it to be constant on the bicharacteristic leaves of $J_{-}$. For $t_{0}$ real, we consider the complex Lagrangian manifold $K_{t_{0}} \subset J_{-}^{\mathbf{C}}$, given by $q_{1}=t_{0}$. This manifold intersects the real domain cleanly along the bicharacteristic leaf $\Gamma_{t_{0}}^{-}$of $J_{-}$, passing through the point of $\gamma^{0}$-corresponding to $t_{0}$. If $q_{2}, \ldots, q_{n}$ are in involution such that $J_{-}$is given by $q_{2}=\ldots=q_{n}=0$, then locally we can extend $q_{1}$ to the full space, so that $q_{1}, q_{2}, \ldots, q_{n}$ are in involution. Then since $q_{2}, \ldots, q_{n}$ are real, we also have: $\left\{\bar{q}_{1}, q_{k}\right\}=\left\{\overline{q_{1}, q_{k}}\right\}=0$. Modulo the directions $H_{q_{2}}, \ldots, H_{q_{n}}$, the field $H_{q_{1}}$ in the sense of the full symplectic structure coincides with $H_{q_{1}}$ in the sense of the symplectic structure of $\bar{\gamma}$. Hence $i^{-1} \sigma\left(H_{q_{1}}, \overline{H_{q_{1}}}\right)$ $=i^{-1}\left\{q_{1}, \overline{q_{1}}\right\}<0$ in the full symplectic structure, and we conclude that $K_{t_{0}}$ is a strictly negative Lagrangian manifold. If we choose $a$ in the definition of $f$ small, then $K_{t_{0}}$ is close to the complex Lagrangian manifold $\left(\Lambda_{-}^{0}\right)^{\mathbf{C}}$.

Our discussion is invariant under the canonical transformation $\kappa_{T}$ introduced in Sect. 1, and if we consider all our objects after this transformation (without changing the notations), then since $K_{t_{0}}$ is close to the Lagrangian manifold $\left(\Lambda_{-}^{0}\right)^{\mathbf{C}}$, we see that the projection $\left.\pi_{x}\right|_{K_{0}}: K_{t_{0}} \rightarrow \mathbf{C}_{x}^{n}$ is a local diffeomorphism near $\gamma_{0}$.

It is easy to see that there exist $C^{\infty}$ sections $v_{2}, \ldots, v_{n}, w_{2}, \ldots, w_{n}$ in $\Gamma\left(\bar{\gamma} ; \mathbf{C}^{n}\right)$ such that at every point $\varrho \in \bar{\gamma}: v_{2}, \ldots, v_{n}$ generate the vectorspace $T_{\varrho}\left(J_{+}^{\mathbf{C}}\right)^{\sigma}$ (the symplectic orthogonal space) and such that $w_{2}, \ldots, w_{n}$ generate $T_{\varrho}\left(J_{-}^{\mathbf{C}}\right)^{\sigma}$. Without changing notation, we now replace $v_{2}, \ldots, v_{n}, w_{2}, \ldots, w_{n}$ by analytic sections which are arbitrarily close in the $C^{\infty}$ topology. Consider the analytic diffeomorphism

$$
\bar{\gamma}^{\mathbf{C}} \times \mathbf{C}^{2(n-1)} \ni(\varrho, t, s) \mapsto \varrho+\sum_{2}^{n} t_{j} v_{j}+\sum_{2}^{n} s_{j} w_{j} \in \mathbf{C}^{2 n},
$$

defined in a neighborhood of $\bar{\gamma} \times\{(0,0)\}$. Then the inverse images of $J_{-}^{\mathbf{c}}$ and $J_{+}^{\mathbf{c}}$ are of the form $t-f(s, \varrho)=0$ and $s-g(t, \varrho)=0$, respectively, where $f$ and $g$ are analytic and well defined near $s=0$ and $t=0$. Back in $\mathbf{C}^{2 n}$, we define $q_{j}$ and $p_{j}$ as the images of $t_{j}-f_{j}$ and $s_{j}-g_{j}$, so that $J_{+}^{\mathbf{c}}$ is given by $p_{2}=\ldots=p_{n}=0$ and $J_{-}^{\mathbf{c}}$ is given by $q_{2}=\ldots=q_{n}=0$. The matrix $\left(\left\{p_{j}, q_{k}\right\}\right)$ is easily seen to be non-degenerate (since $J_{+}$ and $J_{-}$intersect transversally), so we can find a unique non-degenerate analytic matrix $\left(a_{j, k}(\varrho)\right), \varrho \in \bar{\gamma}$, such that if we replace $q_{j}$ by $\sum a_{j, k} q_{k}$, then $\left\{p_{j}, q_{k}\right\}=\delta_{j, k}$ on $\bar{\gamma}$. We shall now improve the choice of $p_{j}, q_{j}$, so that these relations will be valid in a neighborhood of $\bar{\gamma}$. Put $\tilde{q}_{2}=q_{2}$ and let $\Sigma_{1} \supset J_{+}^{\mathbf{C}}$ be the hypersurface $p_{2}=0$, and let $\tilde{q}_{3}$ be the solution of $H_{\tilde{q}_{2}} \tilde{q}_{3}=0$, with initial condition $\left.\tilde{q}_{3}\right|_{\Sigma_{1}}=q_{3}$. Then $d \tilde{q}_{3}=d q_{3}$ on $\bar{\gamma}$, $\tilde{q}_{3}=0$ on $J_{-}$and $H_{\tilde{q}_{2}}, H_{\tilde{q}_{3}}$ commute and are tangent to $J_{-}^{\mathbf{c}}$. Let $\Sigma_{2} \supset J_{+}^{\mathbf{c}}$ be the submanifold; $p_{2}=p_{3}=0$. Let $\tilde{q}_{4}$ be the solution of the well posed problem

$$
H_{\tilde{q}_{2}} \tilde{q}_{4}=H_{\tilde{q}_{3}} \tilde{q}_{4}=0,\left.\quad \tilde{q}_{4}\right|_{\Sigma_{2}}=q_{4} .
$$

Continuing this procedure, we get a new set of functions $\tilde{q}_{2}, \ldots, \tilde{q}_{n}$ keeping all the properties above and such that in addition $\left\{\tilde{q}_{j}, \tilde{q}_{k}\right\}=0$. Finally, improve $p_{2}, \ldots, p_{n}$ by solving

$$
H_{\tilde{q}_{j}} \tilde{p}_{k}=-\delta_{j, k},\left.\quad \tilde{p}_{k}\right|_{J_{-}}=0 .
$$

Since $\left\{p_{j}, p_{k}\right\}=0$ on $J_{+}^{\mathbf{C}}$, these relations hold everywhere, by the Jacobi identities. 
Summing up, we now have $p_{2}, \ldots, p_{n}, q_{2}, \ldots, q_{n}$ satisfying $\left\{p_{j}, p_{k}\right\}=0,\left\{q_{j}, q_{k}\right\}=0$, $\left\{p_{j}, q_{k}\right\}=\delta_{j, k}$. In particular, the corresponding Hamilton fields form a Frobenius system of commuting vector fields, transversal to $\bar{\gamma}$ at every point. We finally extend the functions $p_{1}, q_{1}$ from $\bar{\gamma}$ to a neighborhood, by solving

$$
H_{p_{j}} p_{1}=H_{q_{j}} p_{1}=0, \quad H_{p_{j}} q_{1}=H_{q_{j}} q_{1}=0, \quad 2 \leqq j \leqq n .
$$

(This gives back the extension of $q_{1}$ to $J_{-}$, already discussed.) We have $\left\{p_{1}, q_{1}\right\}=1$ for the symplectic structure of $\bar{\gamma}$, and (2.1) shows that $d p_{1}$ and $d q_{1}$ restricted to the symplectic orthogonal space of $\bar{\gamma}$ vanish. Hence $\left\{p_{1}, q_{1}\right\}=1$ on $\bar{\gamma}$ for the full symplectic structure and from (2.1) and the Jacobi identities, we conclude that $\left\{p_{1}, q_{1}\right\}=1$ everywhere.

Summing up, we have constructed a system of symplectic coordinates $p_{1}, \ldots, p_{n}$, $q_{1}, \ldots, q_{n}$, defined in a complex neighborhood of $\bar{\gamma}$. All the coordinates are singlevalued except $q_{1}$, which satisfies $\mathscr{T} q_{1}=q_{1}+2 \pi$. Let us then consider the canonical transformation,

$$
\kappa:(y, \eta) \mapsto(x, \xi)=(q(y, \eta), p(y, \eta)) \in\left(S^{1} \times i \mathbf{R}\right) \times \mathbf{C}^{n-1} \times \mathbf{C}^{n} .
$$

The image of $\bar{\gamma}^{\mathbf{C}}$ is given by $x^{\prime \prime}=\xi^{\prime \prime}=0$, where we write $x=\left(x_{1}, x^{\prime \prime}\right), \xi=\left(\xi_{1}, \xi^{\prime \prime}\right)$. We also know that $\kappa(\bar{\gamma})$ is an $I$-Lagrangian and $\mathbf{R}$-symplectic submanifold of $\kappa\left(\bar{\gamma}^{\mathbf{C}}\right)$ such that the fibers $x_{1}=$ const are strictly negative with respect to $\kappa(\bar{\gamma})$. Hence we know from [13], that $\kappa(\bar{\gamma})$ is given by: $\xi_{1}=2 i^{-1} \partial_{x_{1}} \widetilde{\Phi}_{1}$, where $\widetilde{\Phi}_{1}=\widetilde{\Phi}_{1}\left(x_{1}\right)$ is strictly subharmonic. Since $\gamma^{0}$ is mapped to $\xi_{1}=0, \operatorname{Im} x_{1}=0$, we also know that $\tilde{\Phi}_{1}\left(x_{1}\right)$ $=O\left(\left|\operatorname{Im} x_{1}\right|^{2}\right)$, and hence $\widetilde{\Phi}_{1}\left(x_{1}\right) \sim\left(\operatorname{Im} x_{1}\right)^{2}$.

From the results in the appendix and the hyperbolic structure of $\gamma^{0}$, it is easy to see that there is an escape function $G$ such that in the region $p^{-1}\left(\left[-\varepsilon_{0}, \varepsilon_{0}\right]\right)$ :

$$
H_{p} G>0 \text { in } C \bar{\gamma} .
$$

$$
\begin{array}{cl}
\text { Near } \bar{\gamma} \text { we have: } & G=O\left(\operatorname{dist}(\cdot, \bar{\gamma})^{2}\right), \\
H_{p} G \sim \operatorname{dist}(\cdot, \bar{\gamma})^{2}, & \left.G\right|_{J_{ \pm}} \sim \pm \operatorname{dist}(\cdot, \bar{\gamma})^{2} .
\end{array}
$$

We can take for example a local escape function $G_{1}$ equivalent to $\operatorname{dist}\left(\Gamma_{-}\right)^{2}$ - $\operatorname{dist}\left(\Gamma_{+}\right)^{2}$, and glue $G_{1}$ to an escape function at infinity $\widetilde{G}$ constructed in Proposition A.6. From now on we consider the situation after applying $\kappa_{T}$ (without change of notation). It is rather easy to see that since for example the bicharacteristic leaves of $J_{-}$map into the fibres $x=$ const, $\kappa\left(\Lambda_{\Phi}\right)$ does not project nicely on the $x$-space, but we shall see that things improve, if we replace $\Phi$ by $\Phi_{t}=\Phi+t G$ for some small $t>0$. Here $G$ is considered also as a function on $\mathbf{C}_{y}^{n}$ in view of the projection $\Lambda_{\Phi} \rightarrow \mathbf{C}_{y}^{n}$.

Remark 2.1. In the original coordinates of $\mathbf{C}^{2 n}$, we may introduce as in [6] the $I$ Lagrangian manifold $\Lambda_{t G}: \operatorname{Im}(x, \xi)=t H_{G}(\operatorname{Re}(x, \xi))$. Then $\kappa_{T}\left(\Lambda_{t G}\right)=\Lambda_{\hat{\Phi}_{t}}$, where with the same identification as above; $\hat{\Phi}_{t}=\Phi+t \hat{G}, \hat{G}=G+O\left(t \operatorname{dist}(\cdot, \bar{\gamma})^{2}\right)$. The discussion below applies also to $\hat{\Phi}_{t}$.

Now let us consider the graph $C$ of $\kappa$, defined in a complex neighborhood of $\left\{\left(\left(x_{1}, 0\right), 0, \gamma^{0}\left(x_{1}\right)\right) ; \operatorname{Im} x_{1}=0\right\}$, where for simplicity we use the variable " $t$ " introduced above as a parametrization for $\gamma^{0}$. If we fix $x=\left(x_{1}, 0\right)$ with $x_{1}$ real, then the inverse image of $\left\{(x, \xi) ; \xi \in \mathbf{C}^{n}\right\}$ under $\kappa$ is the manifold $K_{x_{1}}$ discussed above, 
and we have arranged so that the projection $K_{x_{1}} \rightarrow \mathrm{C}_{y}^{n}$ is a local diffeomorphism. It follows from this, that the map $C \ni(x, \xi, y, \eta) \mapsto(x, y)$ is a local diffeomorphism, so

$$
C=\left\{\left(x, \phi_{x}^{\prime}(x, y), y,-\phi_{y}^{\prime}(x, y)\right)\right\},
$$

where $\phi(x, y)$ is a holomorphic, possibly multivalued function defined near $V=\left\{\left(x_{1}, 0, \pi_{y} \gamma^{0}\left(x_{1}\right)\right)\right\} \subset\left(\left(S^{1} \times i \mathbf{R}\right) \times \mathbf{C}^{n-1}\right) \times \mathbf{C}^{n}$. To study the multivaluedness of $\phi$, it suffices to study $\phi(\mathscr{T} x, \mathscr{T} y)-\phi(x, y)$, for $(x, y) \in V$, where $\mathscr{T}$ denotes the rotation either on $S^{1}$ or in $\pi_{y}\left(\gamma^{0}\right)$. On this particular set we know that $\phi_{x}^{\prime}=0$, $-\phi_{y}^{\prime}=\left(\phi_{+}^{0}\right)_{x}^{\prime}=\left(\phi_{-}^{0}\right)_{x}^{\prime}$, so we conclude from (1.7) that:

$$
\phi(\mathscr{T} x, \mathscr{T} y)=\phi(x, y)-C(0),
$$

when $(x, y) \in V$, and hence also for all $(x, y)$ in a neighborhood of $V$.

We now look for suitable contours, in order to realize a Fourier integral operator $F$ with phase $\phi(x, y)$. Fix $x^{0}=\left(x_{1}, 0\right)$ with $x_{1}$ real. Then $\Lambda_{-\phi\left(x^{0}, \cdot\right)}=K_{x_{1}}$, and if we recall that the real part of $K_{x_{1}}$ is the bicharacteristic leaf $\Gamma_{x_{1}}$ in $J_{-}$, we see that

and that

$$
-\operatorname{Im}\left(-\phi\left(x^{0}, y\right)\right)-\Phi(y)=O\left(\operatorname{dist}\left(y, \pi_{y}\left(\Gamma_{x_{1}}\right)\right)^{2}\right),
$$

$$
-\operatorname{Im}\left(-\phi\left(x^{0}, y\right)\right)-\Phi(y) \sim-\operatorname{dist}\left(y, \pi_{y}\left(\Gamma_{x_{1}}\right)\right)^{2},
$$

on $\pi_{y}\left(\Gamma_{x_{1}}^{\mathbf{c}}\right)$. Now we recall that $K_{x_{1}}$ is a negative Lagrangian manifold, so if we add a suitable direction to $\pi_{y}\left(\Gamma_{x_{1}}\right)$, not in $\pi_{y}\left(\Gamma_{x_{1}}^{\mathbf{C}}\right)$, we get a totally real manifold $L_{x_{1}}$ of dimension $n$, containing $\pi_{y}\left(\Gamma_{x_{1}}\right)$, such that

$$
-\operatorname{Im}\left(-\phi\left(x^{0}, y\right)\right)-\Phi(y) \sim \operatorname{dist}\left(y, \pi_{y}\left(\Gamma_{x_{1}}\right)\right)^{2} \quad \text { on } L_{x_{1}},
$$

or rather,

$$
-\operatorname{Im}\left(\phi\left(x^{0}, y\right)\right)+\Phi(y) \sim-\operatorname{dist}\left(y, \pi_{y}\left(\Gamma_{x_{1}}\right)\right)^{2} \quad \text { on } L_{x_{1}} .
$$

If we replace $\Phi$ by $\Phi_{t}$, we get

$$
-\operatorname{Im}\left(\phi\left(x^{0}, y\right)+\Phi_{t}(y) \sim-\operatorname{dist}\left(y, \pi_{y}\left(\gamma^{0}\left(x_{1}\right)\right)^{2} \quad \text { on } L_{x_{1}} .\right.\right.
$$

This means that if $f(x, y, h)$ is a classical analytic elliptic symbol, defined in a neighborhood of $V$ and if $u \in H_{\Phi_{t}}^{\text {loc }}$ is defined in a neighborhood of $\pi_{y} y^{0}$, then we can define $F u \in H_{\Phi_{t}}^{\text {loc }}$ in a neighborhood of $S^{1} \times\{0\}$, by

$$
F u(x, h)=\int e^{i \phi(x, y) / h} g(x, y, h) v(x) d x .
$$

In this formula we integrate along the contour $L_{x_{1}}$.

Here $\widetilde{\Phi}_{t}$ is strictly plurisubharmonic (pl.s.h.) and $\Lambda_{\tilde{\Phi}_{t}}=\kappa\left(\Lambda_{\Phi_{t}}\right),\left.\widetilde{\Phi}_{t}\right|_{x^{\prime \prime}=0}=\Phi_{1}$, and more precisely $\widetilde{\Phi}_{t}(x)=\widetilde{\Phi}_{1}\left(x_{1}\right)+O\left(\left(x^{\prime \prime}\right)^{2}\right)$. Here we use the terminology and the results of [13]. From [13, Theorem 4.5], we also know that $F$ has an inverse $G$ (which inverts modulo equivalence in the spaces $H_{\Phi_{t}}^{\text {loc }}$ and $H_{\tilde{\Phi}_{t}}^{\text {loc }}$ ), of the form

$$
G v(y, h)=\int e^{-i \phi(x, y) / h} g(x, y, h) v(x) d x .
$$

We have to be aware of one fact: In view of (2.6), $F u$ will not be singlevalued in general, but will satisfy a Floquet periodicity condition:

$$
F u(\mathscr{T} x, h)=e^{-i C(0) / h} F u(x, h) .
$$


We get a new pseudodifferential operator FPG, that by abuse of language, we shall also denote by $P$. Let $p$ also denote the new principal symbol. Both $x^{\prime \prime}=0$ and $\xi^{\prime \prime}=0$ are invariant under the flow of $H_{p}$, which means that

$$
p\left(x, \xi_{1}, 0\right)=p\left(x_{1}, 0, \xi_{1}, 0\right)=p\left(x_{1}, 0, \xi\right) .
$$

If $q\left(x_{1}, \xi_{1}\right)=p\left(x_{1}, 0, \xi_{1}, 0\right)$, we then get

$$
p(x, \xi)=q\left(x_{1}, \xi_{1}\right)+\sum_{2}^{n} \sum_{2}^{n} a_{j, k}(x, \xi) x_{j}^{\prime \prime} \xi_{k}^{\prime \prime} .
$$

It is easy to see that the stable outgoing Lagrangian manifold $\Lambda_{+}^{\varepsilon}$ is strictly positive with respect to $\Lambda_{\Phi_{t}}$, with real part $\gamma^{\varepsilon}$. Now $\kappa$ maps $\gamma^{\varepsilon}$ into $\tilde{\gamma}^{\varepsilon} \subset\left\{x^{\prime \prime}=\xi^{\prime \prime}=0\right\}$, given by $\operatorname{Im} x_{1}=C_{\varepsilon}\left(\operatorname{Re} x_{1}\right), \xi_{1}=(2 / i) \partial_{x_{1}} \widetilde{\Phi}_{1}\left(x_{1}\right)$. The manifold $\tilde{\Lambda}_{+}^{\varepsilon}=\kappa\left(\Lambda_{+}^{\varepsilon}\right)$ is strictly positive with respect to $\Lambda_{\tilde{\Phi}_{t}}$, and generated by $\tilde{\phi}_{+}^{\varepsilon}\left(x_{1}\right)$, where $\tilde{\phi}_{+}^{\varepsilon}$ is a generally multivalued phase function with

$$
\tilde{\Phi}_{t}(x)-\left(-\operatorname{Im} \tilde{\phi}_{+}^{\varepsilon}\left(x_{1}\right)\right) \sim \operatorname{dist}\left(x, \pi\left(\tilde{\gamma}_{\varepsilon}\right)\right)^{2} .
$$

We can specify the multivaluedness:

$$
\tilde{\phi}_{+}^{\varepsilon}\left(\mathscr{T} x_{1}\right)-\tilde{\phi}_{+}^{\varepsilon}\left(x_{1}\right)=C(\varepsilon)-C(0) .
$$

Putting $\varepsilon=0$ in (2.12), we get

$$
\tilde{\Phi}_{t}(x) \sim\left|x^{\prime \prime}\right|^{2}+\left|\operatorname{Im} x_{1}\right|^{2} .
$$

Since $\left.\operatorname{Im} p\right|_{\Lambda_{\Phi_{t}}} \sim-\operatorname{dist}(\cdot, \bar{\gamma})^{2}$ (in a neighborhood of $\gamma$ ), we get after transformation

$$
\left.\operatorname{Im} p\right|_{\Lambda \tilde{\Phi}_{t}} \sim-\left|x^{\prime \prime}\right|^{2}
$$

\section{Estimates for a Grushin Problem in One Dimension}

Let $P\left(x_{1}, \xi_{1}, h\right)$ be a classical analytic symbol of order 0 defined near the zero section of $T^{*}\left(S^{1} \times i \mathbf{R}\right)$ restricted to $S^{1} \times\{0\}$ with values in the $N \times N$ matrices. We assume that $P$ has an asymptotic expansion:

$$
P \sim \sum_{j \in \mathbf{N} / 2} p_{j}(x, \xi) h^{j}
$$

with:

$$
\begin{gathered}
p_{0}\left(x_{1}, \xi_{1}\right) \text { is equal to } q \text { defined in (2.11), } \\
p_{1 / 2}=0 .
\end{gathered}
$$

Recall that it follows from (2.12) and the definition of $\widetilde{\Phi}_{1}\left(x_{1}\right)$ that:

$$
\tilde{\Phi}_{1}\left(x_{1}\right)=-\operatorname{Im}\left(\phi^{\varepsilon}\left(x_{1}\right)\right)+g\left(x_{1}, \varepsilon\right),
$$

where $g\left(x_{1}, \varepsilon\right)$ is equivalent to $\operatorname{dist}\left(x_{1}, \pi_{x_{1}}\left(\gamma^{\varepsilon}\right)\right)^{2}$, and $\phi^{\varepsilon}\left(x_{1}\right)$ is a holomorphic function such that $q_{1}\left(x_{1}, \partial_{x_{1}} \phi^{\varepsilon}\right)=\varepsilon$. Let $x_{1}(\varepsilon) \in \pi_{x_{1}}\left(\gamma^{\varepsilon}\right)$ be the point with $\operatorname{Re} x_{1}(\varepsilon)=0$. We can assume that $\operatorname{Re} \phi^{\varepsilon}\left(x_{1}(\varepsilon)\right)=0$. If $\Omega_{1} \subset \subset \Omega_{2} \subset \subset \Omega_{3}$ are small complex neighborhoods of $S^{1}$, then we have the following proposition: 
Proposition 3.1. We can find operators $R_{-}(\varepsilon): \mathbf{C}^{N} \rightarrow H_{\tilde{\Phi}_{1}}\left(\Omega_{2}\right), R_{+}(\varepsilon): H_{\tilde{\Phi}_{1}}\left(\Omega_{1}\right) \rightarrow \mathbf{C}^{N}$ such that if $\left(u, u_{-}\right) \in H_{\tilde{\Phi}_{1}}\left(\Omega_{3}\right) \times \mathbf{C}^{N}$ satisfies:

$$
\left\{\begin{array}{l}
(i / h)(P-\varepsilon) u+R_{-} u_{-}=v \quad \text { in } \Omega_{2}, \\
R_{+} u=v_{+},
\end{array}\right.
$$

for $\left(v, v_{+}\right) \in H_{\tilde{\Phi}_{1}}\left(\Omega_{2}\right) \times \mathbf{C}^{N}$, and if $u, v$ are $z$-Floquet periodic, then the following estimates hold uniformly for $z$ in a compact annulus of $\mathbf{C} \backslash\{0\}$ :

$$
\begin{gathered}
\|u\|_{\Omega_{1}} \leqq C\left(h^{1 / 4}\left\|v_{+}\right\|+\|v\|_{\Omega_{2}}+O\left(h^{\infty}\right)\left(\|u\|_{\Omega_{3}}+\left\|u_{-}\right\|\right)\right), \\
\left\|u_{-}\right\| \leqq C\left(\left\|v_{+}\right\|+h^{-1 / 4}\|v\|_{\Omega_{2}}+O\left(h^{\infty}\right)\left(\|u\|_{\Omega_{3}}+\left\|u_{-}\right\|\right)\right) .
\end{gathered}
$$

Here $\|u\|_{\Omega_{1}}$ is the norm in $H_{\tilde{\Phi}_{1}}\left(\Omega_{1}\right)$;

$$
\|u\|_{\Omega_{1}}^{2}=\iint_{\Omega_{1}}|u|^{2} e^{-2 \tilde{\Phi}_{1 / h}} L(d x), \quad L(d x)=\text { Lebesgue measure }
$$

Proof. In the following we will often omit " $\varepsilon$ " in the notation. We start by reducing $(i / h) P$ to $\partial_{x_{1}}$. To do this, we construct an elliptic $N \times N$ symbol of order $0 ; a(x, \varepsilon, h)$ such that $(P-\varepsilon)\left(a e^{i \phi / h}\right) \sim 0$, by solving the usual transport equations. If $\mathscr{T}$ is the rotation by $2 \pi$ on $S^{1}$ (or rather its covering space), we get from (2.13):

$$
\phi^{\varepsilon}\left(\mathscr{T} x_{1}\right)=\phi^{\varepsilon}\left(x_{1}\right)+C(\varepsilon)-C(0)
$$

and we also have,

$$
a\left(x_{1}, \varepsilon, h\right)=U(\varepsilon, h) a\left(x_{1}, \varepsilon, h\right),
$$

where $U$ is an elliptical symbol of order 0 . Then we have the following equality between operators:

$$
e^{-i \phi / h} a^{-1}(P-\varepsilon) e^{i \phi / h} a=Q\left(x_{1}, \widetilde{D}_{x_{1}}, h\right) \widetilde{D}_{x_{1}},
$$

where $Q$ is elliptic of order 0 .

Assume now that $\left(u, u_{-}\right) \in H_{\tilde{\Phi}_{1}}\left(\Omega_{3}\right) \times \mathbf{C}^{N}$ satisfies

$$
\left.\begin{array}{l}
(i / h)(P-\varepsilon) u+R_{-} u_{-}=v, \quad\left(v, v_{+}\right) \in H_{\tilde{\Phi}_{1}}\left(\Omega_{2}\right) \times \mathbf{C}^{N}, \\
R_{+} u=v_{+},
\end{array}\right\}
$$

where $u$ and $v$ are assumed to be $z$-Floquet periodic, for $z$ in some compact annulus in $\mathbf{C} \backslash\{0\}$. If $u=e^{i \phi / h} a \hat{u} v=e^{i \phi / h} a \hat{v}$, we get using (3.6):

$$
\left.\begin{array}{l}
Q \partial_{x_{1}} \hat{u}+e^{-i \phi / h} a^{-1} R_{-} u_{-}=\hat{v} \\
R_{+}\left(e^{i \phi / h} a \hat{u}\right)=v_{+} .
\end{array}\right\}
$$

(We here ignore certain exponentially small errors, that will not affect the estimates of the proposition.) Using then (3.4), (3.5) it is easy to see that $\hat{u}$ and $\hat{v}$ are $Z$-Floquet periodic with $Z=z e^{-i(C(\varepsilon)-C(0)) / h} U^{-1}(\varepsilon, h)$. [Moreover, we can easily check that $Q$ will have the right Floquet periodicity to match in (3.8).] We shall now consider $Z$ as a new parameter. Let $y(\varepsilon)$ be a point on $\pi_{x}\left(\gamma^{\varepsilon}\right)$ and let $f\left(x_{1}, \varepsilon\right)$ be the gaussian: $f\left(x_{1}, \varepsilon\right)=\exp \left(-C_{0}\left(x_{1}-y(\varepsilon)\right)^{2}\right)$, where $C_{0}$ is a large positive constant. Then we set

$$
F\left(x_{1}, \varepsilon, Z\right)=\sum_{-\infty}^{+\infty} Z^{-k} f\left(x_{1}+2 \pi k, \varepsilon\right)
$$


which is close to $f\left(x_{1}, \varepsilon\right)$ on the "interval" $\left[x_{1}(\varepsilon), x_{1}(\varepsilon)+2 \pi\right]$, if $C_{0}$ is large enough, but $F$ is now $z$-Floquet periodic.

We consider now the following Grushin problem:

$$
\left.\begin{array}{l}
\partial_{x_{1}} \hat{u}+\hat{R}_{-} u_{-}=\hat{v}, \\
\hat{R}_{+} \hat{u}=v_{+},
\end{array}\right\}
$$

where $\hat{R}_{-} u_{-}=F\left(x_{1}, \varepsilon, Z\right) u_{-}, \hat{R}_{+} \hat{u}=\hat{u}\left(x_{1}(\varepsilon)\right)$. Then the general solution of (3.9) is given by:

$$
\hat{u}\left(x_{1}\right)=v_{+}+\int_{x_{1}(\varepsilon)}^{x_{1}} \hat{v}(t) d t-\int_{x_{1}(\varepsilon)}^{x_{1}} F(t, \varepsilon, Z) u_{-} d t .
$$

Since we are looking for $Z$-Floquet periodic solutions, we get

$$
(1-Z) v_{+}+\int_{x_{1}(\varepsilon)}^{x_{1}(\varepsilon)+2 \pi} \hat{v}(t) d t-\int_{x_{1}(\varepsilon)}^{x_{1}(\varepsilon)+2 \pi} F(t, \varepsilon, Z) u_{-} d t=0 .
$$

Let $G\left(x_{1}, \varepsilon, Z\right)$ be the function:

$$
\int_{x_{1}(\varepsilon)}^{x_{1}} F(t, \varepsilon, Z) d t
$$

It is clear that $G\left(x_{1}(\varepsilon)+2 \pi, \varepsilon, Z\right)$ is invertible, so by composing $f$ with some constant non-vanishing matrix, we can arrange so that $G\left(x_{1}(\varepsilon)+2 \pi, \varepsilon, Z\right)=1$. Then we get

$$
u_{-}=(1-Z) v_{+}+\int_{x_{1}(\varepsilon)}^{x_{1}(\varepsilon)+2 \pi} \hat{v}(t) d t
$$

We need now some estimates on the solution of (3.9), and we start by getting pointwise estimates. Let $x_{0} \in \Omega_{1}$ : Since $g$ is strictly subharmonic, for every $x_{0}$ we can find $\tilde{g}=\operatorname{Re} b, b$ holomorphic, such that: $g-\tilde{g} \sim d\left(x, x_{0}\right)^{2}$. If $\chi \in C_{0}^{\infty}(\mathbf{C})$ is a standard cutoff function, then using the Cauchy integral formula, we get:

so

$$
e^{-2 b\left(x_{0}\right) / h} \hat{v}\left(x_{0}\right)^{2}=\frac{1}{2 \pi i} \int \frac{\hat{v}(z)^{2}}{x_{0}-z} e^{-2 b(z) / h} h^{-1 / 2} \bar{\partial}_{z} \chi\left(\frac{x_{0}-z}{h^{1 / 2}}\right) L(d z),
$$

$$
\begin{aligned}
& \left|\hat{v}\left(x_{0}\right)\right|^{2} e^{-2 g\left(x_{0}\right) / h}=\left|\hat{v}\left(x_{0}\right)^{2} e^{-2 b\left(x_{0}\right) / h}\right| \\
& \quad \leqq C h^{-1} \int|\hat{v}(z)|^{2} e^{-2 \tilde{g}(z) / h}\left|\bar{\partial}_{z} \chi\left(\left(x_{0}-z\right) / h^{1 / 2}\right)\right| L(d z) \\
& \quad=\frac{C}{h} \int|\hat{v}(z)|^{2} e^{-2 g(z) / h} e^{2(g(z)-\tilde{g}(z)) / h}\left|\bar{\partial}_{z} \chi\left(\left(x_{0}-z\right) / h^{1 / 2}\right)\right| L(d z) .
\end{aligned}
$$

Since $g(z)-\tilde{g}(z)=O\left(\left|z-x_{0}\right|^{2}\right), e^{(g-\tilde{g}) / h}$ is bounded by some constant independent of $h$ on the support of $\chi\left(\left(x_{0}-z\right) / h^{1 / 2}\right)$. Finally, we get:

$$
\left|\hat{v}\left(x_{0}\right)^{2} e^{-2 g\left(x_{0}\right) / h}\right| \leqq \frac{C}{h} \int_{\left|x_{0}-z\right| \leqq h^{1 / 2}}|\hat{v}(z)|^{2} e^{-2 g(z) / h} L(d z) .
$$

Integrating this estimate along $\pi_{x}\left(\gamma^{\varepsilon}\right)$, where $g$ vanishes, we get:

$$
\int_{x_{1}(\varepsilon)}^{x_{1}(\varepsilon)+2 \pi}|\hat{v}(t)|^{2} d t \leqq C h^{-1 / 2}\|\hat{v}\|_{\Omega_{1}}^{2},
$$


where $\|\hat{v}\|_{\Omega_{1}}$ denotes the norm in $H_{g}\left(\Omega_{1}\right)$. Using the Cauchy-Schwarz inequality, we then obtain:

$$
\left|\int_{x_{1}(\varepsilon)}^{x_{1}(\varepsilon)+2 \pi} \hat{v}(t) d t\right| \leqq C h^{-1 / 4}\|\hat{v}\|_{\Omega_{1}}^{2} .
$$

This implies that:

$$
\left\|u_{-}\right\| \leqq C\left(\left\|v_{+}\right\|+h^{-1 / 4}\|\hat{v}\|_{\Omega_{1}}\right)
$$

We now estimate

$$
I\left(x_{1}\right)=\int_{x_{1}(\varepsilon)}^{x_{1}} \hat{v}(t) d t \times e^{-g\left(x_{1}\right) / h} .
$$

To do this, we choose first the integration path in the following way: Let $y\left(x_{1}\right) \in \pi_{x_{1}}\left(\gamma^{\varepsilon}\right)$ be the point which is closest to $x_{1}$. We integrate along $\pi_{x_{1}}\left(\gamma^{\varepsilon}\right)$ from $x_{1}(\varepsilon)$ to $y\left(x_{1}\right)$, and then from $y\left(x_{1}\right)$ to $x_{1}$.

Let $I\left(x_{1}\right)=I_{1}\left(x_{1}\right)+I_{2}\left(x_{1}\right)$ be the corresponding decomposition of $I\left(x_{1}\right)$. Using the same estimate as before, we get:

$$
\begin{gathered}
\left|I_{1}\left(x_{1}\right)\right| \leqq e^{-g\left(x_{1}\right) / h} C h^{-1 / 4}\|\hat{v}\|_{\Omega_{1}}, \\
I_{2}\left(x_{1}\right) \leqq \\
\int_{y\left(x_{1}\right)}^{x_{1}} e^{\left(g(t)-g\left(x_{1}\right)\right) / h}\left|e^{-g(t) / h} \hat{v}(t)\right||d t| \\
\leqq\left(\int_{y\left(x_{1}\right)}^{x_{1}} e^{2\left(g(t)-g\left(x_{1}\right)\right) / h}|d t|\right)^{1 / 2} C h^{-1 / 4}\|\hat{v}\|_{\Omega_{1}} .
\end{gathered}
$$

The first factor in the last member has the same behaviour as:

$$
\left(\int_{0}^{x} e^{-\left(x^{2}-t^{2}\right) / h} d t\right)^{1 / 2} \quad(\text { for } x \in \mathbf{R}),
$$

which can easily be estimated by a constant times $h^{1 / 4}$. So, finally: $\left|I_{2}\left(x_{1}\right)\right| \leqq C\|\hat{v}\|_{\Omega_{1}}$.

Integrating the pointwise estimate of $I_{1}\left(x_{1}\right)$, we then get:

$$
\left\|\int_{x_{1}(\varepsilon)}^{x_{1}} \hat{v}(t) d t\right\|_{\Omega} \leqq C\|\hat{v}\|_{\Omega_{1}} .
$$

On the other hand, since $G\left(x_{1}, \varepsilon, Z\right)$ is bounded; $\|G\|_{\Omega_{1}} \leqq C h^{1 / 4}$, so we get the estimate for $\hat{u}$ :

$$
\|\hat{u}\|_{\Omega} \leqq C\left(h^{1 / 4}\left\|v_{+}\right\|+\|\hat{v}\|_{\Omega_{1}}\right) \text {. }
$$

Now we return to the original problem (3.8). Let $Q^{-1}$ be a parametrix for $Q$ so that $Q^{-1} Q=1-R_{-\infty}$, where $R_{-\infty}$ has an operator norm $=O\left(h^{\infty}\right)$ (from the space of $H_{g}$-functions on a domain to the same space on a slightly smaller domain). Note that $\hat{R}_{+} \hat{u}=e^{-i \phi\left(x_{1}(\varepsilon)\right) / h} u\left(x_{1}(\varepsilon)\right)$, since we can choose $a$ such that $a\left(x_{1}(\varepsilon), h\right)=\mathbf{1}$. Applying $Q^{-1}$ to (3.8) gives:

$$
\left\{\begin{array}{l}
\partial_{x_{1}} \hat{u}+\hat{R}_{-} u_{-}=Q^{-1} \hat{v}+R_{\infty} u+R_{-\infty} u_{-}, \\
\hat{R}_{+} \hat{u}=v_{+}
\end{array}\right.
$$

if we use the estimates (3.11), (3.12) and the fact that $R_{\infty}$ and $R_{-\infty}$ are $O\left(h^{\infty}\right)$, we get the proposition. 


\section{Solution of a Grushin Problem on $\mathbf{R}^{\boldsymbol{n}}$}

Composing the standard F.B.I.-transform mentioned in Sect. 1 with the integral transform constructed in Sect. 2, we get an operator of norm $O(1)$ :

$$
T: H\left(\Lambda_{t G}, 1\right) \rightarrow H_{\Phi}(\Omega) .
$$

Here $t>0$ is small and fixed, $G$ is the escape function introduced in Sect. 2 and $\Phi$ denotes a function having all the properties of $\tilde{\Phi}_{t}$ in Sect. 2. (See Remark 2.1.) In (4.1), $\Omega$ is a small open neighborhood of $S^{1} \times\{0\}$ in $\left(S^{1} \times i \mathbf{R}\right) \times \mathbf{C}^{n-1}$, and $H_{\Phi}(\Omega)$ denotes the space of holomorphic functions in $\Omega$ such that

$$
\|u\|_{H}^{2}=\int|u(x)|^{2} e^{-2 \Phi(x) / h} L(d x)<\infty .
$$

By convention the elements of $H_{\Phi}(\Omega)$ are not single valued but $\omega$-Floquet periodic as in (2.9), with $\omega=e^{-i C(0) / h}$. (Other choices of $\omega$ will also be made.) The direct definition of $F$ in Sect. 2, only gives that $\bar{\partial} T u$ is exponentially small, but we can correct this by solving a $\bar{\partial}$-problem, using the fact that $\Phi$ is strictly plurisubharmonic.

$T$ has microlocal inverse $S$ of norm $O(1): H_{\Phi}(\Omega) \rightarrow H\left(\Lambda_{t G}, m\right)$ with the properties:

(i) $S T$ is a pseudodifferential operator of order 0 adapted to $\Lambda_{t G}$ in the sense of [6], which has compactly supported symbol and which realizes the identity microlocally near $\bar{\gamma}$.

(ii) We have $\|T S u-u\|_{H_{\Phi}(\tilde{\Omega})}=O\left(h^{\infty}\right)\|u\|_{H_{\Phi}(\Omega)}$, where $\widetilde{\Omega} \subset C \Omega$ has the same properties as $\Omega$.

As we also discussed in Sect. 2, our original operator $P$ transforms into a pseudodifferential operator, that we shall also denote by $P$ (and sometimes also by $\widetilde{P}$, when both operators are considered simultaneously). As we know from [13], there are several ways of realizing $P$, and they are all essentially equivalent. In this section, we make essential use of the techniques of [11] and [13], and we shall not recall all details. Since the weightfunction $\Phi$ is fixed from now on, we shall suppress it from our notation as much as possible, and we write $H(\Omega)$ or $H^{0}(\Omega)$ instead of $H_{\Phi}(\Omega)$.

We introduce the following weighted spaces:

$$
H^{m}(\Omega)=\left\{u \in H(\Omega) ;\left(1+h^{-1 / 2}\left|x^{\prime \prime}\right|\right)^{m} u \in L^{2}\left(\Omega, e^{-2 \Phi / h} L(d x)\right)\right\},
$$

with their natural norms. All these spaces are equal to $H^{0}(\Omega)$, and the importance is entirely due to the weighted norm

$$
\|u\|_{H^{m}}=\left\|\left(1+h^{-1 / 2}\left|x^{\prime \prime}\right|\right)^{m} u\right\|_{L^{2}\left(\Omega, e^{-2 \Phi / h} L(d x)\right)} .
$$

Let $A(x, \xi, h)$ be an analytic symbol defined in a neighborhood of $\left\{\left(x,(2 / i) \partial_{x} \Phi(x)\right) ; x \in \bar{\Omega}\right\}$, such that

$$
A(x, \xi, h)=O(1)\left(1+h^{-1 / 2}\left(\left|x^{\prime \prime}\right|+\left|\xi^{\prime \prime}\right|\right)\right)^{m_{0}} .
$$

By " $A$ " we also denote any reasonable realization $A(x, \tilde{D}, h): H\left(\Omega_{2}\right) \rightarrow H\left(\Omega_{1}\right)$, where $\Omega_{1} \subset \subset \Omega_{2} \subset \subset \Omega$. Then (cf. [11, (2.17)]), we have

Proposition 4.1. $A$ is $O(1), h \rightarrow 0$, as a bounded operator $H^{m}\left(\Omega_{2}\right) \rightarrow H^{m-m_{0}}\left(\Omega_{1}\right)$.

The proof is merely a repetition of the arguments in [11], so we shall omit it. 
Corollary 4.2. The operator $\left(h^{-1 / 2} x^{\prime \prime}\right)^{\alpha}\left(h^{1 / 2} D_{x^{\prime \prime}}\right)^{\beta}$ is $O(1): H^{m}\left(\Omega_{2}\right) \rightarrow H^{m-|\alpha|-|\beta|}\left(\Omega_{1}\right)$.

From now on, we only consider domains $\Omega$ such that for some $\varepsilon_{0}>0$ : $\Omega \cap\left\{\left|x^{\prime \prime}\right|<\varepsilon_{0}\right\}=\Omega^{\prime} \times\left\{x^{\prime \prime} ;\left|x^{\prime \prime}\right|<\varepsilon_{0}\right\}, \Omega^{\prime}=\left\{x_{1} ;\left(x_{1}, 0\right) \in \Omega\right\}$. The main purpose of this section is to perform a reduction of the study of $P$, to the study of a onedimensional pseudodifferential operator, acting on truncated Taylor expansions in $x^{\prime \prime}$ at $x^{\prime \prime}=0$. For $N \in \mathbf{N}$, we introduce the expansions:

$$
\begin{aligned}
\tau_{N} u(x) & =\sum_{\left|\alpha^{\prime \prime}\right|<N}(\alpha !)^{-1}\left(\partial_{x^{\prime \prime}}^{\alpha} u\right)\left(x_{1}, 0\right)\left(x^{\prime \prime}\right)^{\alpha} \\
& =\sum_{\left|\alpha^{\prime \prime}\right|<N}(\alpha !)^{-1}\left(\left(h^{1 / 2} \partial_{x^{\prime \prime}}\right)^{\alpha} u\right)\left(x_{1}, 0\right)\left(h^{-1 / 2} x^{\prime \prime}\right)^{\alpha} .
\end{aligned}
$$

We recall that our weight $\Phi(x)$ satisfies $\Phi(x)-\Phi\left(x_{1}, 0\right) \sim\left|x^{\prime \prime}\right|^{2}$, and we shall write $\Phi_{1}=\left.\Phi\right|_{x^{\prime \prime}=0}$.

Proposition 4.3. If $\Omega_{1} \subset \subset \Omega_{2} \subset \subset \Omega$, then for all $m_{1}, m_{2}$ the operator $\tau_{N}$ is $O(1): H^{m_{2}}\left(\Omega_{2}\right) \rightarrow H^{m_{1}}\left(\Omega_{1}\right)$.

Proof. Let $d=n-1$. Let $\chi \in C_{0}^{\infty}\left(\mathbf{C}^{d}\right)$ be rotation invariant with $\int \chi L\left(d x^{\prime \prime}\right)=1$. If $u$ is holomorphic, then by the mean value property

$$
u\left(x_{1}, 0\right)=h^{-d} \int \chi\left(h^{-1 / 2} x^{\prime \prime}\right)(x) L\left(d x^{\prime \prime}\right) .
$$

Hence, by Cauchy-Schwartz' inequality:

$$
\left\|u\left(x_{1}, 0\right)\right\|_{L^{2}\left(\Omega_{j}^{\prime}\right)} \leqq C h^{-d / 2}\|u\|_{L^{2}\left(\Omega_{j}\right)},
$$

where all $L^{2}$-norms are evaluated either with respect to $e^{-2 \Phi / h} L(d x)$ or with respect to $e^{-2 \Phi_{1} / h} L\left(d x_{1}\right)$, unless otherwise is explicitly stated. Since we integrate over

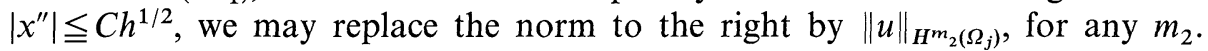
Conversely, if $v\left(x_{1}\right) \in H\left(\Omega_{j}^{\prime}\right)$, then

$$
\left\|v\left(x_{1}\right)\left(h^{-1 / 2} x^{\prime \prime}\right)^{\alpha}\right\|_{L^{2}\left(\Omega_{j}\right)} \leqq C h^{d / 2}\|v\|_{L^{2}\left(\Omega_{j}^{\prime}\right)},
$$

and we even have

$$
\left\|v\left(x_{1}\right)\left(h^{-1 / 2} x^{\prime \prime}\right)^{\alpha}\right\|_{H^{m_{1}\left(\Omega_{j}\right)}} \leqq C\|v\|_{L^{2}\left(\Omega_{j}^{\prime}\right)} .
$$

Combining this with Corollary 4.2, we get the result.

To make our reduction to the study of an operator on Taylor expansions, we shall first prove a "strong" a priori estimate for functions that vanish to some high but fixed order at $x^{\prime \prime}=0$. The first step will be to establish a half estimate, using a method inspired from Cordoba-Fefferman [3].

Proposition 4.4. Let $\chi \in C_{0}^{\infty}(\Omega)$, and choose a realization of $P$ such that $P u$ is welldefined in a neighborhood of supp $\chi$, when $u \in H(\Omega)$. Then there is a classical $C^{\infty}$ symbol $q(x, h)$ of order 0 with support contained in $\operatorname{supp} \chi$, such that

$$
\begin{aligned}
& \int P u(x, h) \overline{v(x, h)} \chi(x) e^{-2 \Phi(x) / h} L(d x) \\
& \quad=\int q(x, h) u(x, h) \overline{v(x, h)} e^{-2 \Phi(x) / h} L(d x)+r(u, v),
\end{aligned}
$$

where $|r(u, v)| \leqq C_{N} h^{N}\|u\| \times\|v\|$ for all $N$, and the principal part of $q($ modulo $O(h))$ is given by

$$
q_{0}(x)=p\left(x, \frac{2}{i} \partial_{x} \Phi(x)\right) \chi(x) .
$$


Proof. Using analytic stationary phase (see [13]), we first notice that in a neighborhood $W$ of supp $\chi$ :

$$
P u(x, h)=\left.\sum_{|\alpha| \leqq \varepsilon_{1} / h} \frac{1}{\alpha !} P^{(\alpha)}\left(x, \frac{2}{i} \partial_{x} \Phi\right)\left(\tilde{D}_{y}-\frac{2}{i} \partial_{x} \Phi(x)\right)^{\alpha} u(y)\right|_{y=x}+R u(x, h),(4
$$

where

$$
\|R u\|_{L^{2}(\Omega)} \leqq e^{-\varepsilon_{2} / h}\|u\|
$$

We can then ignore the contribution from $R u$. We have

$$
\left.\left(\tilde{D}_{y}-\frac{2}{i} \partial_{x} \Phi(x)\right)^{\alpha} u(y)\right|_{y=x}=\sum_{\beta \leqq \alpha} h^{1 / 2|\alpha-\beta|} f_{\alpha, \beta}(x, h)\left(\tilde{D}_{x}-\frac{2}{i} \partial_{x} \Phi\right)^{\beta} u(x),
$$

where $f_{\alpha, \beta}=O(1), f_{\alpha, \alpha}=1$ (and where $\frac{1}{2}$-integer powers of $h$ are actually absent).

Insert this with (4.11) in the left-hand side of (4.9) and integrate by parts. Then (4.9) follows. [Notice that no derivative can fall on $\overline{v(x)}$ which is anti-holomorphic.] The leading term $q_{0}(x)$ is produced from the leading term in (4.11) and (4.10) follows.

Now take $\Omega_{1} \subset \subset \Omega_{2} \subset \subset \Omega_{3} \subset \subset \Omega$, and let $\chi \in C_{0}^{\infty}\left(\Omega_{2}\right)$ take its values in $[0,1]$ and be equal to 1 on $\Omega_{1}$. We also assume that the realization of $P$ is chosen so that $P u$ is well defined in $\Omega_{2}$, for $u \in H\left(\Omega_{3}\right)$. From (2.15) and Proposition 4.4, we get

$$
h\|u\|_{H^{1}\left(\Omega_{1}\right)}^{2} \leqq C\left(-\operatorname{Im}(P u \mid \chi u)+h\|u\|_{H^{1}\left(\Omega_{3} \backslash \Omega_{1}\right)}^{2}+h\|u\|_{H^{0}\left(\Omega_{1} \cap\left\{\left|x^{\prime \prime}\right| \leqq C h^{1 / 2}\right\}\right)}^{2}\right),
$$

for all $u \in H\left(\Omega_{3}\right)$.

Let $O^{N}$ denote the space of holomorphic functions $u$ such that $u=O_{h}\left(\left|x^{\prime \prime}\right|^{N}\right)$. We shall now see, that if $N \in \mathbf{N}$ is sufficiently large, then we can eliminate the last term in (4.12), when $u \in H\left(\Omega_{3}\right) \cap O^{N}$.

Lemma 4.5. Let $0<C_{0}<C_{1}$. Then for $u \in H\left(\Omega_{1}\right) \cap O^{N}$ :

$$
\|u\|_{\left\{\left|x^{\prime \prime}\right| \leqq C_{0} h^{1 / 2}\right\} \cap \Omega_{1}} \leqq C(N)^{-1}\|u\|_{\left\{\left|x^{\prime \prime}\right| \leqq C_{1} h^{1 / 2}\right\}_{\cap} \Omega_{1}},
$$

where $C(N) \rightarrow \infty, N \rightarrow \infty$.

Proof. It is enough to prove the corresponding inequality for the $L^{2}$-norms with $x_{1}$ fixed. The variation of $x^{\prime \prime} \mapsto \Phi(x) / h$ is then $O(1)$, so after the change of variables $x^{\prime \prime}=h^{1 / 2} \tilde{x}^{\prime \prime}$, we are then reduced to prove

$$
\|u\|_{L^{2}\left(B\left(0, C_{0}\right)\right)} \leqq \widetilde{C}(N)^{-1}\|u\|_{L^{2}\left(B\left(0, C_{1}\right)\right)},
$$

for all holomorphic functions on the ball $B\left(0, C_{1}\right) \subset \mathbf{C}^{n-1}$ (of center 0 and radius $C_{1}$ ), vanishing to the order $N$ at the origin. Here, of course, we should also have $\widetilde{C}(N) \rightarrow \infty, N \rightarrow \infty$, but the $L^{2}$-norms are now taken with respect to the Lebesgue measure.

After increasing $C_{0}$ and decreasing $C_{1}$ it is enough to prove a corresponding inequality for $L^{\infty}$-norms, with the constant $\widetilde{C}(N)$ slightly changed.

Since $u\left(x^{\prime \prime}\right)$ vanishes to the order $N$ at 0 , we can use Taylor's formula:

$$
u\left(x^{\prime \prime}\right)=\int_{0}^{1} \frac{(1-t)^{N-1}}{(N-1) !}\left(\frac{d}{d t}\right)^{N} u\left(t x^{\prime \prime}\right) d t
$$


We may assume that $\left|x^{\prime \prime}\right|=C_{0}$, and use Cauchy's inequalities directly on $t \rightarrow u\left(t x^{\prime \prime}\right)$ :

$$
\left|\left(\frac{d}{d t}\right)^{N} u\left(t x^{\prime \prime}\right)\right| \leqq N !\left(C_{1} / C_{0}-t\right)^{-N}\|u\|_{L^{\infty}\left(B\left(0, C_{1}\right)\right)}
$$

Then,

$$
\|u\|_{L^{\infty}\left(B\left(0, C_{0}\right)\right)} \leqq N \int_{0}^{1}(1-t)^{N-1} /\left(C_{1} / C_{0}-t\right)^{N} d t \times\|u\|_{L^{\infty}\left(B\left(0, C_{1}\right)\right)},
$$

and the coefficient to the right can be estimated by $N\left(C_{1} / C_{0}-1\right)^{-1}\left(C_{0} / C_{1}\right)^{N-1}$ which tends to 0 , as $N$ tends to infinity.

If we fix $N$ sufficiently large, then for $u \in H\left(\Omega_{3}\right) \cap O^{N}$, the last term of (4.12) can be eliminated. (We have to increase the constant $C$.) We use also that

$$
-\operatorname{Im}(P u \mid \chi u) \leqq\|P u\|_{H^{-1}\left(\Omega_{2}\right)}\|u\|_{H^{1}\left(\Omega_{3}\right)},
$$

and get (with a new constant $C$ ):

$$
h\|u\|_{H^{1}\left(\Omega_{1}\right)}^{2} \leqq C\left(\|P u\|_{H^{-1}\left(\Omega_{2}\right)}\|u\|_{H^{1}\left(\Omega_{3}\right)}+h\|u\|_{H^{1}\left(\Omega_{3} \backslash \Omega_{1}\right)}^{2}\right),
$$

which implies

$$
h\|u\|_{H^{1}\left(\Omega_{3}\right)}^{2} \leqq C\left(\|P u\|_{H^{1}\left(\Omega_{3}\right)}\|u\|_{H^{1}\left(\Omega_{3}\right)}+h\|u\|_{H^{1}\left(\Omega_{3} \backslash \Omega_{1}\right)}\|u\|_{H^{1}\left(\Omega_{3}\right)} .\right.
$$

Dividing by $\|u\|_{H^{1}\left(\Omega_{3}\right)}$, we get

Proposition 4.6. Let $\Omega_{1} \subset \subset \Omega_{2} \subset \subset \Omega_{3} \subset \subset \Omega$. If $N \in \mathbf{N}$ is sufficiently large, then for all $u \in H\left(\Omega_{3}\right) \cap O^{N}$ :

$$
h\|u\|_{H^{1}\left(\Omega_{3}\right)} \leqq C\left(\|P u\|_{H^{-1}\left(\Omega_{2}\right)}+h\|u\|_{H^{1}\left(\Omega_{3} \backslash \Omega_{1}\right)}\right) .
$$

By the same proof we also get (4.14) uniformly with $P$ replaced by $P-E$, where $E \in\left[-\varepsilon_{0}, \varepsilon_{0}\right]-i\left[0, C_{0} h\right]$. Also, if we apply this inequality with $u$ replaced by $h^{-1 / 2} x^{\prime \prime} u$, and notice that the commutator $\left[P, h^{-1 / 2} x^{\prime \prime}\right]$ behaves like $h^{1 / 2} x^{\prime \prime}$, we see that we can raise the indices by 1 and get

$$
h\|u\|_{H^{2}\left(\Omega_{3}\right)} \leqq C\left(\|(P-E) u\|_{H^{0}\left(\Omega_{2}\right)}+h\|u\|_{H^{2}\left(\Omega_{3} \backslash \Omega_{1}\right)}\right),
$$

$u \in H\left(\Omega_{3}\right) \cap O^{N}$.

We shall next analyze various compositions between $P, \tau_{N}, 1-\tau_{N}$. We let $\mathscr{T}^{N}$ be the space of holomorphic functions of the form $\sum_{|\alpha|<N} v_{\alpha}\left(x_{1}\right)\left(h^{-1 / 2} x^{\prime \prime}\right)^{\alpha}$. It is the image of $\tau_{N}$. We may decompose elements of $\mathscr{T}^{N}$ into sums of homogeneous polynomials in $x^{\prime \prime}: \mathscr{T}^{N}=\bigoplus_{0}^{\oplus} \mathscr{T}_{\text {hom }}^{k}$. The operator $P^{(N)}=\tau_{N} P \tau_{N}$ can then be represented by a matrix $\left(P_{j, k}^{(N)}\right)_{0 \leqq j, k \leqq N-1}$.

Let [cf. (2.11)]:

$$
P_{0}=q\left(x_{1}, \widetilde{D}_{x_{1}}\right)+\sum_{2}^{n} \sum_{2}^{n} a_{j, k}\left(x_{1}, 0, \tilde{D}_{x_{1}}, 0\right) x_{k}^{\prime \prime} \tilde{D}_{x_{j}^{\prime \prime}}+h p_{-1}\left(x_{1}, 0, \widetilde{D}_{x_{1}}, 0\right),
$$

where $P \sim p+h p_{-1}+\ldots$ Then $P_{0}$ contributes with a diagonal part to $P^{(N)}$ :

$$
\begin{gathered}
P_{0}=\left(\left(P_{0}\right)_{j, k}\right), \quad\left(P_{0}\right)_{j, k}=0, \quad j \neq k, \\
\left(P_{0}\right)_{j, j}=q\left(x_{1}, \widetilde{D}_{x_{1}}\right)+h R_{j}\left(x_{1}, \widetilde{D}_{x_{1}}, h\right),
\end{gathered}
$$


where $R_{j}$ is a (matrix-valued) pseudodifferential operator of order 0 . The problem of analyzing $P^{(N)}$ is then reduced to that of analyzing $\tau_{N}\left(P-P_{0}\right) \tau_{N}$.

Proposition 4.7. We can represent $\tau_{N}\left(P-P_{0}\right) \tau_{N}$ as a matrix of classical pseudodifferential operators $\left(M_{j, k}\left(x_{1}, \tilde{D}_{x_{1}}, h\right)\right)$, where $M_{j, k}$ is of order $-(1+|j-k| / 2)$ for $j \neq k$ and of order $\leqq-2$ for $j=k$.

Proof. It is quite easy to see that $M_{j, k}$ are classical pseudodifferential operators of order $\leqq 0$. For instance, with $Q=P-P_{0}$, let us consider

$$
\begin{aligned}
& Q\left(x, \tilde{D}_{x}, h\right)\left(v\left(x_{1}\right)\left(h^{-1 / 2} x^{\prime \prime}\right)^{\gamma}\right) \\
& \quad=\sum \sum \frac{1}{\alpha !} \frac{1}{\beta !} \partial_{x^{\prime \prime}}^{\alpha} \partial_{\xi^{\prime \prime}}^{\beta} Q\left(x_{1}, 0, \tilde{D}_{x_{1}}, 0, h\right) v\left(x_{1}\right)\left(x^{\prime \prime}\right)^{\alpha}\left(\tilde{D}_{x^{\prime \prime}}^{\beta}\right)\left(h^{-1 / 2} x^{\prime \prime}\right)^{\gamma} .
\end{aligned}
$$

For the study of $\tau_{N} Q \tau_{N}$, we may restrict $\alpha, \beta, \gamma$ to the region $|\alpha|<N,|\beta|<N,|\gamma|<N$. We can then rewrite our expression as a finite sum of terms

$$
C_{\alpha, \beta} \partial_{x^{\prime \prime}}^{\alpha} \partial_{\xi^{\prime \prime}}^{\beta} Q\left(x_{1}, 0, \tilde{D}_{x_{1}}, 0, h\right)\left(v\left(x_{1}\right)\right) h^{|\beta|-|\gamma| / 2}\left(x^{\prime \prime}\right)^{\alpha+\gamma-\beta},
$$

$\beta \leqq \gamma$. In our case, we may split $Q$ into one term which is independent of $h$ but $O\left(\left|x^{\prime \prime}\right|\left|\xi^{\prime \prime}\right|\left(\left|x^{\prime \prime}\right|+\left|\xi^{\prime \prime}\right|\right)\right.$ and one term which is of order -1 and $O\left(\left|x^{\prime \prime}\right|+\left|\xi^{\prime \prime}\right|\right)$ and finally, one term which is of order -2 . We get a contribution to $M_{j, k}$, when $|\alpha+\gamma-\beta|=j$ and $|\gamma|=k$. To get the order correctly, we should rewrite:

$$
h^{|\beta|-|\gamma| / 2}\left(x^{\prime \prime}\right)^{\alpha+\gamma-\beta}=h^{(|\alpha|+|\beta|) / 2}\left(h^{-1 / 2} x^{\prime \prime}\right)^{\alpha+\gamma-\beta},
$$

so the order is $-\frac{1}{2}(|\alpha|+|\beta|)$ plus the order of $Q$. Now $j-k=|\alpha|-|\beta|$. In the first case we also know that $|\alpha| \geqq 1,|\beta| \geqq 1,|\alpha|+|\beta| \geqq 3$, so we get the order $-\frac{1}{2}(|\alpha|+|\beta|)$ $\leqq-\frac{1}{2}|j-k|-\min (|\alpha|,|\beta|)$, which gives the desired estimate. In the other cases, the order is $\leqq-\frac{1}{2}|j-k|-\min (|\alpha|,|\beta|)+$ the order of $Q$, and in each case we get the desired estimate.

We also need,

Proposition 4.8. If $0<M \leqq N$, then $\tau_{M} P\left(1-\tau_{N}\right)=\tau_{M}\left(P-P_{0}\right)\left(1-\tau_{N}\right)$ and $\left(1-\tau_{N}\right) P \tau_{M}=\left(1-\tau_{N}\right)\left(P-P_{0}\right) \tau_{M}$ are $O\left(h^{1 / 2(3+N-M)}\right.$ as bounded operators $H^{m_{2}}\left(\Omega_{2}\right) \rightarrow H^{m_{1}}\left(\Omega_{1}\right)$. Here $\Omega_{1} \subset \subset \Omega_{2} \subset \subset \Omega$ and $m_{1}, m_{2}$ are arbitrary.

Proof. Again we consider more generally $\tau_{M} Q\left(1-\tau_{N}\right)$. If $Q(x, \xi, h) \sim \sum_{0}^{\infty} q_{j}(x, \xi) h^{j}$ with $q_{j}=O\left(\left(\left|x^{\prime \prime}\right|+\left|\xi^{\prime \prime}\right|\right)^{(m-2 j)_{+}}\right)$, then from Propositions 4.1 and 4.3 it follows that $\tau_{M} Q$ and $Q \tau_{M}$ are $O\left(h^{m / 2}\right)$ from $H^{m_{2}}\left(\Omega_{2}\right)$ to $H^{m_{1}}\left(\Omega_{1}\right)$. This means that by a Taylor expansion of the symbol of $Q$ at $x^{\prime \prime}=\xi^{\prime \prime}=0$, we can reduce our problem to the case when $Q$ is differential in the $x^{\prime \prime}$-coordinates. For $|\alpha|<M$ and $u \in O^{N}$, we get

$$
\begin{aligned}
\left(h^{1 / 2} D_{x^{\prime \prime}}\right)^{\alpha}(Q u)\left(x_{1}, 0\right)= & \sum_{\substack{\alpha_{1}+\alpha_{2}=\alpha \\
\beta}} C_{\alpha_{1}+\alpha_{2}, \beta} Q_{\left(\alpha_{1}\right)}^{(\beta)}\left(x_{1}, 0, \widetilde{D}_{x_{1}}, 0, h\right) \\
& \times h^{\left|\alpha_{1}+\beta\right| / 2}\left(\left(h^{1 / 2} D_{x^{\prime \prime}}\right)^{\alpha_{2}+\beta} u\right)\left(x_{1}, 0\right),
\end{aligned}
$$

where the sum is finite. If we consider for instance the case when $Q=q$ is independent of $h$ and $=O\left(\left|x^{\prime \prime}\right|\left|\xi^{\prime \prime}\right|\left(\left|x^{\prime \prime}\right|+\left|\xi^{\prime \prime}\right|\right)\right)$, we can restrict the sum to $\left|\alpha_{1}\right|,|\beta| \geqq 1$, $\left|\alpha_{1}\right|+|\beta| \geqq 3,|\beta|+\left|\alpha_{2}\right| \geqq N,\left|\alpha_{1}\right|+\left|\alpha_{2}\right| \leqq M-1$. The general term in the sum repre- 
sents a term which is $O\left(h^{1 / 2\left(\left|\alpha_{1}\right|+|\beta|\right)}\right)$ as a bounded operator $H^{m_{2}}\left(\Omega_{2}\right) \rightarrow H^{m_{1}}\left(\Omega_{1}\right)$. It is easy to see that $\frac{1}{2}\left(\left|\alpha_{1}\right|+|\beta|\right) \geqq \frac{1}{2}(N-M+3)$. The lower order contributions to $Q$ are treated the same way, and we get the proposition for $\tau_{M} Q\left(1-\tau_{N}\right)$, $Q=P-P_{0}$.

To prove the same statement for $\left(1-\tau_{N}\right) Q \tau_{M}$, we choose $|\alpha|<M$ and consider

$$
Q\left(v\left(x_{1}\right)\left(\left(h^{-1 / 2} x^{\prime \prime}\right)^{\alpha}\right)=\sum_{\beta \leqq \alpha} C_{\alpha, \beta} Q^{(\beta)}\left(x, \tilde{D}_{x_{1}}, 0, h\right)\left(v\left(x_{1}\right)\right) h^{\frac{1}{2}|\beta|}\left(h^{-1 / 2} x^{\prime \prime}\right)^{\alpha-\beta} .\right.
$$

Write,

$$
Q^{(\beta)}\left(x, \xi_{1}, 0, h\right)=\sum_{|\alpha|<k}(\alpha !)^{-1} Q_{(\alpha)}^{(\beta)}\left(x_{1}, 0, \xi_{1}, 0, h\right)\left(x^{\prime \prime}\right)^{\alpha}+R_{k}^{(\beta)}\left(x, \xi_{1}, 0, h\right),
$$

where $R_{k}^{(\beta)}$ vanishes to the order $k$ at $x^{\prime \prime}=0$. Then

$$
\begin{aligned}
(1- & \left.\tau_{N}\right) Q\left(v\left(h^{-1 / 2} x^{\prime \prime}\right)^{\alpha}\right) \\
= & \sum_{\beta \leqq \alpha} C_{\alpha, \beta} R_{N-|\alpha-\beta|}^{(\beta)}\left(x, \widetilde{D}_{x_{1}}, 0, h\right)\left(v\left(x_{1}\right)\right) h^{\frac{1}{2}|\beta|}\left(h^{-1 / 2} x^{\prime \prime}\right)^{\alpha-\beta} \\
= & \sum_{\beta \leqq \alpha} C_{\alpha, \beta} h^{|\beta|+\frac{1}{2}(N-|\alpha|)}\left[h^{-\frac{1}{2}(N-|\alpha-\beta|)}\right. \\
& \left.\times R_{N-|\alpha-\beta|}^{(\beta)}\left(x, \widetilde{D}_{x_{1}}, 0, h\right)\left(v\left(x_{1}\right)\right)\left(h^{-1 / 2} x^{\prime \prime}\right)^{\alpha-\beta}\right] .
\end{aligned}
$$

Here the factor $[\ldots]$ represents an operator on $v\left(x_{1}\right)\left(h^{-1 / 2} x^{\prime \prime}\right)^{\alpha}$ which is $O(1): H^{m_{2}}\left(\Omega_{2}\right) \rightarrow H^{m_{1}}\left(\Omega_{1}\right)$, so the only problem is to find a lower bound for $|\beta|+\frac{1}{2}(N-|\alpha|)$. Considering again the three different cases, we get the desired boundedness. [In the first case, we have $|\beta|+\frac{1}{2}(N-|\alpha|) \geqq 3 / 2+\frac{1}{2}(N-M)$.]

We identify a function

$$
u=\sum_{|\alpha|<N} u_{\alpha}\left(x_{1}, h\right)\left(h^{-1 / 2} x^{\prime \prime}\right)^{\alpha}
$$

in $\mathscr{T}^{N}$ with the $C^{M}$-valued function $\hat{u}\left(x_{1}, h\right)=\left(u_{\alpha}\right)_{|\alpha| \leqq N}$. Here $M=\#\left\{\alpha \in \mathbf{N}^{n-1}\right.$; $|\alpha|<N\}$. We shall say that $\Omega$ is of product type, if near $x^{\prime \prime}=0, \Omega$ is given by conditions on $x_{1}$ only. If $\Omega$ is of product type and if $\Omega^{\prime}=\Omega \cap\left\{x^{\prime \prime}=0\right\}$, then we have uniform equivalence between $\|u\|_{H^{m}(\Omega)}$ and $h^{(n-1) / 2}\|\hat{u}\|_{H_{\tilde{\Phi}_{1}\left(\Omega^{\prime}\right)} \text {. The operator } P^{(N)}}$ satisfies all the assumptions of Sect. 3. Let $S^{1} \times\{0\} \subset \subset \Omega_{1} \subset \subset \Omega_{2} \subset \subset \Omega_{3} \subset \subset \Omega$ be of product type:

Proposition 4.9. For $u \in H^{2}\left(\Omega_{3}\right), v \in H^{0}\left(\Omega_{2}\right), u_{-}, v_{+} \in \mathbf{C}^{M}$, we consider the Grushin problem:

$$
\left.\begin{array}{l}
(i / h)(P-E) u+R_{-}^{N} u_{-}=v \quad \text { in } \Omega_{2} \\
R_{+}^{N} u=v_{+}
\end{array}\right\}
$$

where $R_{+}^{N}, R_{-}^{N}$ are adapted to $P^{(N)}$ as in Sect. 3. Here we let $u, v$ be $z$-Floquet periodic, where $z$ varies in a compact annulus in $\mathbf{C} \backslash\{0\}$. Then uniformly in $u, u_{-}, z$, we have with $\tilde{n}=2 n-1$

$$
\left(\|u\|_{H^{2}\left(\Omega_{1}\right)}+h^{\tilde{n} / 4}\left\|u_{-}\right\|\right) \leqq C\left(\|v\|_{H^{2}\left(\Omega_{2}\right)}+h^{\tilde{n} / 4}\left\|v_{+}\right\|+O\left(h^{\infty}\right)\|u\|_{H^{2}\left(\Omega_{3}\right)}\right) .
$$

Proof. Applying $\left(1-\tau_{N}\right)$ to the first equation of (4.17), we get

$$
(i / h)\left(1-\tau_{N}\right)(P-E) u=\left(1-\tau_{N}\right) v,
$$


which implies

$$
(i / h)(P-E)\left(1-\tau_{N}\right) u=\left(1-\tau_{N}\right) v+\frac{i}{h}\left(\tau_{N}(P-E)\left(1-\tau_{N}\right) u-\left(1-\tau_{N}\right)(P-E) \tau_{N} u\right) .
$$

Using (4.15) and Proposition 4.8, we get,

$$
\left\|\left(1-\tau_{N}\right) u\right\|_{H^{2}\left(\Omega_{1}\right)} \leqq C\left(\|v\|_{H^{0}\left(\Omega_{2}\right)}+h^{1 / 2}\|u\|_{H^{2}\left(\Omega_{3}\right)}+\|u\|_{H^{2}\left(\Omega_{3} \backslash \Omega_{1}\right)}\right) .
$$

Applying also $\tau_{N}$ to (4.17), we get

$$
\left\{\begin{array}{l}
(i / h) \tau_{N}(P-E) u+R_{-}^{N} u_{-}=\tau_{N} v \\
R_{+}^{N} \tau_{N} u=v_{+}
\end{array}\right.
$$

since $\tau_{N} R_{-}^{N}=R_{-}^{N}, R_{+}^{N}=R_{+}^{N} \tau_{N}$. Then,

$$
(i / h) \tau_{N}(P-E) \tau_{N} u+R_{-}^{N} u_{-}=\tau_{N} v-(i / h) \tau_{N}(P-E)\left(1-\tau_{N}\right) u,
$$

so using the estimates of Propositions 3.1 and 4.8 and the remark prior to Proposition 4.9, we get

$$
\left(\left\|\tau_{N} u\right\|_{H^{2}\left(\Omega_{1}\right)}+h^{\tilde{n} / 4}\left\|u_{-}\right\|\right) \leqq C\left(\|v\|_{H^{\mathrm{o}}\left(\Omega_{2}\right)}+h^{\tilde{n} / 4}\left\|v_{+}\right\|+h^{1 / 2}\|u\|_{H^{2}\left(\Omega_{3}\right)}\right) .
$$

Adding (4.19) and (4.20), we get,

$$
\left(\|u\|_{H^{2}\left(\Omega_{1}\right)}+h^{\tilde{n} / 4}\left\|u_{-}\right\|\right) \leqq C\left(\|v\|_{H^{0}\left(\Omega_{2}\right)}+h^{\tilde{n} / 4}\left\|v_{+}\right\|+\|u\|_{H^{2}\left(\Omega_{3} \backslash \Omega_{1}\right)}\right) .
$$

In the above estimates, we now replace $\Omega_{2}$ and $\Omega_{3}$ by $\widetilde{\Omega}_{2}, \widetilde{\Omega}_{3}$ with $\Omega_{1} \subset \subset \widetilde{\Omega}_{2}$ CC $\widetilde{\Omega}_{3} \subset C \Omega_{2}$. Since $P-E$ is elliptic in a neighborhood of $\widetilde{\Omega}_{3} \backslash \Omega_{1}$, we have

$$
\|u\|_{H^{2}\left(\tilde{\Omega}_{3} \backslash \Omega_{1}\right)} \leqq C\left(\|v\|_{H^{0}\left(\Omega_{2}\right)}+O\left(h^{\infty}\right)\|u\|_{H^{2}\left(\Omega_{2}\right)}\right),
$$

so using this in (4.21) (where $\Omega_{3}$ had been replaced by $\widetilde{\Omega}_{3}$ ), we get (4.18).

We now return to the original problem on $\mathbf{R}^{n}$. Let $\chi\left(x, \widetilde{D}_{x}, h\right)$ be a pseudodifferential operator of order 0 adapted to $\Lambda_{t G}$ with compactly supported symbol, equal to the identity microlocally near $\bar{\gamma}$ and with the property that $\chi(I-S T)$ and $(I-S T) \chi$ are negligible (in the sense of [6, Chap. 6]). Here $T$ and $S$ are the Fourier integral operators introduced in the beginning of this section. We put $\hat{R}_{+}=R_{+} T \chi$, $\hat{R}_{-}=S R_{-}$, where $R_{ \pm}=R_{ \pm}^{N}$, and where we take the natural Floquet periodicity; $z=\exp (-i C(0) / h)$. Then we have the following theorem:

Theorem 4.10. For $h>0$ small enough and $E \in\left[-\varepsilon_{0}, \varepsilon_{0}\right]+i\left[0,-C_{0} h\right]$, the Grushin problem,

$$
\left.\begin{array}{l}
(i / h)(P-E) u+\hat{R}_{-} u_{-}=v, \\
\hat{R}_{+} u=v_{+},
\end{array}\right\}
$$

has a unique solution $\left(u, u_{-}\right) \in H\left(\Lambda_{t G}, m\right) \times \mathbf{C}^{M}$ for every $\left(v, v_{+}\right) \in H\left(\Lambda_{t G}, 1\right) \times \mathbf{C}^{M}$. Moreover, we have the a priori estimate

$$
\left(\|u\|_{H\left(\Lambda_{t G}, m\right)}+h^{\tilde{n} / 4}\left\|u_{-}\right\|\right) \leqq C\left(\|v\|_{H\left(\Lambda_{t G}, 1\right)}+h^{\tilde{n} / 4}\left\|v_{+}\right\|\right),
$$

where $C$ is independent of $E$ and $\tilde{n}=2 n-1$.

Proof. Since $\left.(p-E)\right|_{\Lambda_{t G}}$ is elliptic outside a small neighborhood of $\gamma^{E}$, it follows from the general theory of [6], that

$$
\|(1-\chi) u\|_{H\left(\Lambda_{t G}, m\right)} \leqq C\left(\|v\|_{H\left(\Lambda_{t G}, 1\right)}+O\left(h^{\infty}\right)\left(\|u\|_{H\left(\Lambda_{t G}, m\right)}+\left\|u_{-}\right\|\right)\right) .
$$


Here we also use that $\hat{R}_{-} u_{-}$is $O\left(h^{\infty}\right)$ in $H\left(\Lambda_{t G}, 1\right)$ outside $\gamma^{E}$.

Applying $\chi\left(x, \widetilde{D}_{x}, h\right)$ to the first equation in (4.22), we get

$$
\left\{\begin{array}{l}
(i / h)(P-E) \chi u+\chi \hat{R}_{-} u_{-}=\chi v+w, \\
R_{+} T \chi u=v_{+}
\end{array}\right.
$$

where $w=(i / h)[P, \chi] u$ satisfies:

$$
\|w\|_{H\left(A_{t G}, 1\right)} \leqq C\left(\|v\|_{H\left(A_{t G}, 1\right)}+O\left(h^{\infty}\right)\left(\|u\|_{H\left(A_{t G}, m\right)}+\left\|u_{-}\right\|\right)\right) .
$$

Here we use (4.24) with a cutoff closer to $\gamma^{E}$. If $\tilde{u}=T \chi u$, and if $\widetilde{P}$ is a realization of the conjugated operator TPS, we get,

$$
\left\{\begin{array}{l}
(i / h)(\tilde{P}-E) \tilde{u}+T \hat{R}_{-} u_{-}=T(\chi v+w)+\tilde{w}, \\
R_{+} \tilde{u}=v_{+},
\end{array}\right.
$$

where $\|\tilde{w}\|_{H^{0}\left(\Omega_{3}\right)}=O\left(h^{\infty}\right)\|u\|_{H\left(\Lambda_{t G}, m\right) \cdot}$. Here $\Omega_{1}, \Omega_{2}, \Omega_{3}$ are as in Proposition 4.9, and we may assume that $\Omega_{1}$ or rather $\kappa_{T}^{-1}\left(\left.\Lambda_{\Phi}\right|_{\Omega_{1}}\right)$ is much larger than the region where $\chi$ is not $\equiv 0$ microlocally. Here

$$
T \hat{R}_{-} u_{-}=T S R_{-} u_{-}=R_{-} u_{-}-\tilde{w}_{-},
$$

where $\left\|\tilde{w}_{-}\right\|_{H^{0}\left(\Omega_{3}\right)}=O\left(h^{\infty}\right)\left\|u_{-}\right\|$. If we write,

$$
\left\{\begin{array}{l}
(i / h)(\tilde{P}-E) \tilde{u}+R_{-} u_{-}=T(\chi v+w)+\tilde{w}+\tilde{w}_{-}, \\
R_{+} \tilde{u}=v_{+},
\end{array}\right.
$$

we can apply Proposition 4.9 and get after using the inverse transform:

$$
\begin{aligned}
& \|\chi u\|_{H\left(\Lambda_{t G}, m\right)}+h^{\tilde{n} / 4}\left\|u_{-}\right\| \\
& \quad \leqq C\left(\|v\|_{H\left(\Lambda_{t G}, 1\right)}+h^{\tilde{n} / 4}\left\|v_{+}\right\|+O\left(h^{\infty}\right)\left(\|u\|_{H\left(\Lambda_{t G}, m\right)}+\left\|u_{-}\right\|\right)\right) .
\end{aligned}
$$

Adding (4.24) and (4.25), we get (4.23). Thus we have also proved that

$$
\mathscr{P}=\left(\begin{array}{cc}
(i / h)(P-E) & R_{-} \\
\hat{R}_{+} & 0
\end{array}\right): H\left(\Lambda_{t G}, m\right) \times \mathbf{C}^{M} \rightarrow H\left(\Lambda_{t G}, 1\right) \times \mathbf{C}^{M}
$$

is injective. From the general results of [6], we know that $(P-E)$ and hence also $\left(\begin{array}{cc}(i / h)(P-E) & 0 \\ 0 & 0\end{array}\right)$ are Fredholm of index 0 . Since $\left(\begin{array}{cc}0 & \hat{R}_{-} \\ \hat{R}_{+} & 0\end{array}\right)$ is of finite rank, it follows that $\mathscr{P}$ is also Fredholm of index 0 and hence bijective, since it is injective.

\section{Proof of Theorem 2}

Let $\mathscr{F}=\left(\begin{array}{ll}F & F_{+} \\ F_{-} & F_{-+}\end{array}\right)$be the inverse of $\mathscr{P}$ constructed in Theorem 4.10. We shall first compute an asymptotic expansion for $F_{-+}$. To do this, we need to determine $u_{-}$as a function of $v_{+}$in the problem

$$
\left.\begin{array}{l}
(i / h)(P-E) u+\hat{R}_{-} u_{-}=0, \\
\hat{R}_{+} u=v_{+}
\end{array}\right\}
$$


Then it suffices to get an approximate solution of the problem with errors $O\left(h^{\infty}\right)\left\|v_{+}\right\|$. This asymptotic problem is most easily treated after applying the transformation $T$ :

$$
\left.\begin{array}{l}
(i / h)(\tilde{P}-E) \tilde{u}+R_{-} u_{-}=O\left(h^{\infty}\right), \\
R_{+} \tilde{u}=v_{+}+O\left(h^{\infty}\right) .
\end{array}\right\}
$$

We shall look for $u$ of the form $b(x, E, h) e^{i \phi_{+}(x, E) / h}$, where $b$ is an analytic symbol. Then by applying $S$ to $\tilde{u}$, we will get a solution of (5.1) modulo $O\left(h^{\infty}\right)$ in $H\left(\Lambda_{t_{G}}, 1\right) \times \mathbf{C}^{M}$. To describe properly the structure of $F_{-+}$, we shall first consider (5.2) for $z e^{i(C(E)-C(0)) / h}$ Floquet periodic solutions and afterwards put $z=e^{-i C(E) / h}$. We now denote $\widetilde{P}$ simply by $P$. We shall assume that $E$ lies in the basic rectangle $D=\left[-\varepsilon_{0}, \varepsilon_{0}\right] \times\left[0,-C_{0} h\right]$ for a fixed constant $C_{0}$, and that $N$ is so large that

$$
\text { For }|\alpha|>N ; \quad\left|\varrho(E) \theta(E)^{-\alpha}-e^{-i C(E) / h}\right| \geqq C_{1}>0 .
$$

Let

$$
L=(i / h) e^{-i \phi / h}(P-E) e^{i \phi / h},
$$

and let $S^{m, k}$ be the space of symbols $a(x, h)$ having asymptotic expansions,

$$
a(x, h) \sim \sum_{j=0}^{\infty} h^{-(m-j / 2)} a_{k-j}(x),
$$

where $a_{k-j}(x)=O\left(\left|x^{\prime \prime}\right|^{k-j}\right)$ is holomorphic and $z$-Floquet periodic in $x_{1}$. If $l(x, \xi)$ is the principal symbol of $(h / i) L$, we see that

$$
l(x, \xi)=l_{1}\left(x_{1}, \xi_{1}\right)+B(x, \xi) x^{\prime \prime} \cdot \xi^{\prime \prime},
$$

where $l_{1}=O\left(\left|\xi_{1}\right|\right)$.

We note that $S^{m, k} / S^{m, k+1}$ can be identified with the space of functions $\sum_{j=0}^{k} h^{(m-j / 2)} a_{k-j}(x)$, where $a_{k-j}$ is a $(k-j)$-homogeneous polynomial in the $x^{\prime \prime}-$ variables. Using (5.4) it is easy to verify that $S^{m, k}$ is stable under the action of $L$ (defined by formal asymptotic expansions). The action of $L$ on $S^{m, k} / S^{m, k+1}$ is given by

$$
\mathscr{L}=\sum_{j=1}^{n}\left(\partial_{\xi_{j}} l\right) \partial_{x_{j}}+l_{-1}
$$

or by

$$
\mathscr{L}_{0}=\partial_{\xi_{1}} l_{1}\left(x_{1}, 0\right) \partial_{x_{1}}+B\left(x_{1}, 0,0\right) x^{\prime \prime} \cdot \partial_{x^{\prime \prime}}+l_{-1}\left(x_{1}, 0,0\right) .
$$

We shall then solve the Grushin problem

$$
\left.\begin{array}{l}
L b+a Q F\left(x_{1}, Z\right) u_{-}=c, \\
\left(\left.\left(h^{1 / 2} \widetilde{D}_{x^{\prime \prime}}\right)^{\alpha} b\left(x_{1}(E)\right)\right|_{x^{\prime \prime}=0}\right)_{|\alpha|<N}=v_{+}
\end{array}\right\}
$$

for $b, c \in S^{m, N}$. Here $a, Q, F$ are defined in Sect. 3 for $P=P^{(N)}$. We return for a while to the constructions there. Let $a_{0}, q_{0}$ denote the principal symbols of $a$ and $Q$. With the notations of Sect. 4, it is clear, using Proposition 4.7, that to compute $a_{0}$, we can 
replace $P^{(N)}$ by

$$
\begin{aligned}
P_{0} & =q\left(x_{1}, \tilde{D}_{x_{1}}\right)+\sum_{2}^{n} \sum_{2}^{n} a_{j, k}\left(x_{1}, 0, \tilde{D}_{x_{1}}, 0\right) x_{j}^{\prime \prime} \widetilde{D}_{x_{k}^{\prime \prime}}+h p_{-1}\left(x_{1}, 0, \widetilde{D}_{x_{1}}, 0\right) \\
& =q\left(x_{1}, \widetilde{D}_{x_{1}}\right)+\frac{h}{i} M\left(x_{1}, \widetilde{D}_{x_{1}}\right)+h p_{-1} .
\end{aligned}
$$

Then $a_{0}$ satisfies the transport equation

$$
\frac{\partial q}{\partial \xi_{1}}\left(x_{1}, \frac{\partial \phi_{+}}{\partial x_{1}}\right) \frac{\partial a_{0}}{\partial x_{1}}+\left(M\left(x_{1}, \frac{\partial \phi_{+}}{\partial x_{1}}\right)+i p_{-1}+\frac{1}{2} \frac{\partial^{2} q}{\partial \xi^{2}} \frac{\partial^{2} \phi_{+}}{\partial x_{1}^{2}}\right) a_{0}=0 .
$$

Since conjugation by $e^{i \phi_{+} / h}$ corresponds to the canonical transformation $\left(x_{1}, \xi_{1}\right) \mapsto\left(x_{1}, \xi_{1}-\partial_{x_{1}} \phi_{+}\right)$, we see that $l\left(x_{1}, \xi_{1}\right)=q\left(x_{1}, \partial_{x_{1}} \phi_{+}+\xi_{1}\right), B\left(x_{1}, 0,0\right)$ $=\left(a_{j, k}\left(x_{1}, 0, \partial_{x_{1}} \phi, 0\right)\right), l_{-1}\left(x_{1}, 0,0\right)=i p_{-1}\left(x_{1}, 0, \partial_{x_{1}} \phi_{+}\right)+\frac{1}{2} \partial_{\xi_{1}}^{2} q \partial_{x_{1}}^{2} \phi_{+}$, so one has $\mathscr{L}_{0} a_{0}=0$. We also note that $\partial_{\xi_{1}} l_{1}\left(x_{1}, 0\right)$ is equal to $q_{0}\left(x_{1}, 0\right)$, if $q_{0}$ is the principal symbol of the operator $Q$ introduced in (3.6). Now we come back to (5.5) and we start by studying this problem with $b, c \in S^{m, N} / S^{m, N+1}$. We see that we can replace $a$ by $a_{0}, Q$ by $q_{0}\left(x_{1}, 0\right)$, since we get then an error term in $S^{m-\frac{1}{2}, N} C S^{m, N+1}$.

With the interpretation of $S^{m, N} / S^{m, N+1}$ above, we can obtain an $h$-independent problem (" $h=1$ ") by putting $x^{\prime \prime}=h^{1 / 2} y^{\prime \prime}$;

$$
\left.\begin{array}{l}
\mathscr{L}_{0} b+a_{0} q_{0}\left(x_{1}, 0\right) F\left(x_{1}, Z\right) u_{-}=c, \\
b\left(x_{1}(E)\right)=v_{+} .
\end{array}\right\}
$$

Let $M(t, s)$ be the fundamental solution-matrix of $q_{0}^{-1} \mathscr{L}_{0}$, considered as an ordinary differential operator in $x_{1}$. The solution of (5.6) is then:

$$
\begin{aligned}
b\left(x_{1}\right)= & M\left(x_{1}, x_{1}(E)\right) v_{+}+\int_{x_{1}(E)}^{x_{1}} M\left(x_{1}, y_{1}\right) q_{0}^{-1} c\left(y_{1}\right) d y_{1} \\
& -\int_{x_{1}(E)}^{x_{1}} M\left(x_{1}, y_{1}\right) a_{0}\left(y_{1}\right) F\left(y_{1}, Z\right) u_{-} d y_{1} .
\end{aligned}
$$

Now using the remark made above and the fact that $a_{0}\left(x_{1}(E)\right)=1$, we get that: $a_{0}\left(y_{1}\right)=M\left(y_{1}, x_{1}(E)\right)$, so

$$
\begin{aligned}
& \int_{x_{1}(E)}^{x_{1}(E)+2 \pi} M\left(x_{1}(E)+2 \pi, y_{1}\right) a_{0}\left(y_{1}\right) F\left(y_{1}, Z\right) d y_{1} \\
& \quad=\int_{x_{1}(E)}^{x_{1}(E)+2 \pi} M\left(x_{1}(E)+2 \pi, x_{1}(E)\right) F\left(y_{1}, Z\right) d y_{1} \\
& =M\left(x_{1}(E)+2 \pi, x_{1}(E)\right)=U_{0}(E),
\end{aligned}
$$

with the notations of (3.5). Since $b\left(x_{1}\right)$ is $z$-Floquet periodic, we get:

$$
M\left(x_{1}(E)+2 \pi, x_{1}(E)\right) v_{+}-z v_{+}+\int_{x_{1}(E)}^{x_{1}(E)+2 \pi} M\left(x_{1}(E), y_{1}\right) q_{0}^{-1} c\left(y_{1}\right) d y_{1}=U_{0}(E) u_{-},
$$

so: $E_{-+}^{0} v_{+}=U_{0}(E)^{-1}\left(U_{0}(E)-z\right) v_{+}$. We remark here that the eigenvalues of $U_{0}$ are of the form $\varrho(E) \theta_{1}^{-\alpha_{1}} \ldots \theta_{n-1}^{-\alpha_{n-1}}$, where $\theta_{j}=\theta_{j}(E)$ are the eigenvalues of modulus greater than 1 of the linearized Poincare map associated to $\gamma^{E}$. 
We have then proved that for $c \in S^{N / 2, N}, v_{+} \in\left(S^{0}\right)^{M}$, we can find $b \in S^{N / 2, N}$, $u_{-} \in\left(S^{0}\right)^{M}$, such that

$$
\left\{\begin{array}{l}
L b+a Q F u_{-}=c+d, \\
\left(\left(h^{1 / 2} D_{x^{\prime \prime}}\right)^{\alpha} b\left(x_{1}(E)\right)\right)_{|\alpha|<N}=v_{+},
\end{array}\right.
$$

with $d \in S^{N / 2, N+1}$. We now look for $f \in S^{N / 2, N+1}$ of the form $f=h^{-N / 2} f_{N+1}(x)$, where $f_{N+1}=O\left(\left|x^{\prime \prime}\right|^{N+1}\right)$ is a holomorphic function, such that $L f=d \bmod S^{N / 2-\frac{1}{2}, N}$. Equivalently, we have to solve $\mathscr{L} f=d$. Using (5.3), we see that we can find such a function, if $z$ stays in a fixed neighborhood of $\left\{e^{-i C(E) / h} ; E \in D\right\}$. Finally, we have proved that if $c \in S^{N / 2, N}, v_{+} \in\left(S^{0}\right)^{M}$, we can find $b \in S^{N / 2, N}, u_{-} \in\left(S^{0}\right)^{M}$ such that:

$$
\left\{\begin{array}{l}
L b+a Q F u_{-}=c+d\left(c, v_{+}\right), \\
\left(\left(h^{1 / 2} D_{x^{\prime \prime}}\right)^{\alpha} b\left(x_{1}(E)\right)\right)_{|\alpha|<N}=v_{+},
\end{array}\right.
$$

with $d \in S^{(N-1) / 2, N}$. We can now iterate this procedure, to solve (5.5) for $c=0$. We get that $u_{-}=E_{-+} v_{+}$, with $E_{-+} \in S^{0}$ (here denoting the space of classical symbols with asymptotic expansions in powers of $h^{1 / 2}$ ). The principal part of $E_{-+}$is $E_{-+}^{0}=\left(U^{0}(E)-z\right) U^{0}(E)^{-1}$. We have then proved the following result:

Proposition 5.1. $F_{-+}$is of the form $E_{-+}\left(E, e^{-i C(E) / h}, h\right)+O\left(h^{\infty}\right)$, where $E_{-+} \in S^{0}$ has an asymptotic expansion

$$
E_{-+}(E, z, h) \sim \sum_{0}^{\infty} E_{-+}^{j}(E, z) h^{j},
$$

and where

$$
E_{-+}^{0}=\left(U^{0}(E)-z\right) U^{0}(E)^{-1} .
$$

We are now able to prove Theorem 2 (which implies Theorem 1). Let $\gamma=\gamma(h)$ be simple loop such that $F_{-+}(E, h)$ is invertible for $z \in \gamma$. We shall prove that the number of resonances of $P$ inside $\gamma$ counted with multiplicity is equal to the number of roots inside $\gamma$ of $\operatorname{det} F_{-+}(E, h)=0$. Without changing $F_{-+}$, we can forget the factor $i / h$ in Theorem 4.10 . The first number is equal to

$$
n(\gamma)=(2 \pi i)^{-1} \operatorname{tr} \int_{\gamma}(z-P)^{-1} d z
$$

in view of the general results of [6]. Now

$$
(z-P)^{-1}=-F(z)+F_{+}\left(F_{-+}\right)^{-1} F_{-},
$$

and since $F(z)$ has no singularities inside $\gamma$ and since $F_{+}, F_{-}, F_{-+}$are operators of finite rank, we get

$$
n(\gamma)=(2 \pi i)^{-1} \int_{\gamma} \operatorname{tr}\left(F_{+}\left(F_{-+}\right)^{-1} F_{-}\right)(z) d z .
$$

On the other hand, we know that the number of roots of $\operatorname{det} F_{-+}$inside $\gamma$ is equal to:

$$
m(\gamma)=(2 \pi i)^{-1} \int\left(\operatorname{det} F_{-+}\right)^{-1}\left(\operatorname{det} F_{-+}\right)^{\cdot} d z=(2 \pi i)^{-1} \int \operatorname{tr}\left(\dot{F}_{-+} F_{-+}^{-1}\right) d z
$$


where the dot denotes derivative with respect to $z$. Using that $\dot{\mathscr{F}}=-\mathscr{F} \dot{\mathscr{P}} \mathscr{F}$, we easily get, $\dot{F}_{-+}=F_{-} F_{+}-F_{-+} \dot{R}_{+} F_{+}-F_{-} \dot{R}_{-} F_{-+}$, so

$$
\begin{aligned}
m(\gamma) & =(2 \pi i)^{-1} \int \operatorname{tr}\left(F_{-} F_{+} F_{-+}^{-1}\right)-\operatorname{tr}\left(F_{-} \dot{R}_{-}\right)-\operatorname{tr}\left(F_{-} \dot{R}_{+} F_{+} F_{-+}^{-1}\right) d z \\
& =(2 \pi i)^{-1} \int \operatorname{tr}\left(F_{+} F_{-+}^{-1} F_{-}\right)-\operatorname{tr}\left(F_{-} \dot{R}_{-}\right)-\operatorname{tr}\left(\dot{R}_{+} F_{+}\right) d z \\
& =n(\gamma),
\end{aligned}
$$

since $F_{-} \dot{R}_{-}$and $\dot{R}_{+} F_{+}$have no singularities inside $\gamma$, and therefore do not contribute to the integral.

Now $F_{-+}(E, h)$ is asymptotic to the matrix $E_{-+}\left(E, e^{-i C(E) / h}, h\right)$, defined in Proposition 5.1, and it is easy to see that there is a bijection $b(h)$ between the roots of $\operatorname{det} F_{-+}(E, h)=0$ and the roots of $\operatorname{det} E_{-+}\left(E, e^{-i C(E) / h}, h\right)=0$, such that $b(h) \mu-\mu$ $=O\left(h^{\infty}\right)$. We have then proved the theorem.

Remark 5.2. Modulo $O\left(h^{1 / 2}\right)$, both $E_{-+}$and $F_{-+}^{\infty}=E_{-+} U^{0}(E)$ are of block diagonal form. If $N_{0}<N$ and the last $N-N_{0}$ entries have bounded inverses, it is easy to see that the Grushin problem with the corresponding smaller number of conditions and co-conditions is well posed and that Theorem 2 is still valid. Then, if (5.3) holds with $N$ replaced by $N-1$, Theorem 2 holds. To describe the resonances close to the elements of $\Gamma^{0}(h)$, given by $\alpha=0$ in $(0.13)$, it is enough to take $N=1$. Then $F_{-+}^{\infty}$ is scalar valued, and one can show that only integer powers of $h$ appear in the asymptotic expansion (0.16).

\section{Appendix}

We discuss some general geometric facts, including the existence of suitable escape functions. Some parts of our discussion is inspired from the geometric scattering theory, as it is presented in Reed-Simon [10], but since our potentials may be quite large near infinity, we are far from the situation of ordinary scattering theory, and we therefore give a self-contained discussion.

Let $p(x, \xi) \in S(m)$ be the principal symbol of an operator $P$, which satisfies all the general assumptions of $[6$, Sect. 8], in order to define resonances near 0 . Here $1 \leqq m \in S(m)$. In particular, we assume the existence of an escape function $G \in \dot{S}^{1,1}$, such that

$$
H_{p} G \geqq C^{-1} m \quad \text { on } \quad p^{-1}(0) \backslash K,
$$

where $K$ is a compact set. For our discussion, it will be convenient to have a vector field, which can be integrated for all times, so we introduce $v=m^{-1} H_{p}$. Then the $x$ component $v_{x}$ of $v$ is in the class $S^{-1,0}$ and hence $=O\left(\tilde{r}^{-1}\right)=O\left(r^{-1}\right)$. We recall here that $r(x), R(x)$ are the basic weight functions introduced in [6], and that $\tilde{r}(x, \xi)$ $=\left(r(x)^{2}+\xi^{2}\right)^{1 / 2}$. The time $T$, needed for $(x(t), \xi(t))=\exp t v(x(0), \xi(0))$ to reach a point $(x(T), \xi(T))$, with $|x(T)-x(0)|=\varepsilon R(x(0))$ satisfies the estimate, $T$ const $/ R \geqq \varepsilon R$, so $T \geqq(\varepsilon /$ const $) r R \geqq \varepsilon /$ const. However, to reach infinity, we have to cross infinitely many such time intervals, so it follows that $v$ is integrable for all times.

Notice that (A.1) becomes

$$
v(G) \geqq C^{-1} \quad \text { on } \quad p^{-1}(0) \backslash K .
$$

Here, we may replace $p^{-1}(0)$ by $\Sigma_{p}^{\varepsilon}=p^{-1}([-\varepsilon, \varepsilon])$, if $\varepsilon>0$ is small enough. Indeed, $v(G)$ is of class $S^{0,0}$, so if $\delta>0$ is small enough but independent of $\varrho \in p^{-1}(0) \backslash K$, we 
get $v(G)(\mu) \geqq 1 / 2 C$, for all $\mu \in B(\varrho, \delta)=\left\{(x, \xi) ;\left|x-\varrho_{x}\right|<\delta R\left(\varrho_{x}\right),\left|\xi-\varrho_{\xi}\right|<\delta \tilde{r}(\varrho)\right\}$. Outside the union of all such boxes with $\varrho \in p^{-1}(0)$, we have $|p|>\varepsilon$, for some $\varepsilon>0$. Thus

$$
v(G) \geqq 1 / \hat{C} \quad \text { on } \quad \Sigma_{p}^{\varepsilon} \backslash \tilde{K},
$$

where $\hat{C}>0$ and $\tilde{K}$ is compact. From now on, the work on $\Sigma_{p}^{\varepsilon}$, but most of the discussion will be valid also on $\Sigma_{p}=p^{-1}(0)$.

In view of (A.3), and since the flow of $v$ is complete, we have $G(\varrho(t)) \rightarrow \pm \infty$, $t \rightarrow \pm \infty$, if $\varrho(t)$ is an integral curve for $v$ in $\Sigma_{p}^{\varepsilon} \backslash \tilde{K}$. Choose $T>0$ large enough, so that

$$
\tilde{K} \subset\left\{\varrho \in \Sigma_{p}^{\varepsilon} ;-T+1<G(\varrho)<T-1\right\} .
$$

Let us define the outgoing tail $\Gamma_{+}=\left\{\varrho \in \Sigma_{p}^{\varepsilon}\right.$; $\left.\exp t v(\varrho) \nrightarrow \infty, t \rightarrow-\infty\right\}$. If $\varrho \in \Sigma_{p}^{\varepsilon}$ and $\exp t v(\varrho)$ reaches the region $G \leqq-T+1$ for some negative $t$, then $\varrho$ is not in $\Gamma_{+}$. Hence $\Gamma_{+}$is a closed set contained in the region $G>-T+1$, invariant under the $v$-flow (and hence also under the $H_{p}$-flow).

Proposition A.1. $\Gamma_{+} \cap\{G \leqq s\}$ is compact for each $s>-T+1$.

Proof. This intersection is clearly closed, so we only have to prove that it is bounded. Since our flow is complete, there is a compact set $\hat{K} \subset \Sigma_{p}^{\varepsilon}$, such that $\varrho \in \tilde{K}$, $|t| \leqq(s-(-T+1)) \hat{C} \Rightarrow \exp t v(\varrho) \in \hat{K}$. If then $\mu \in \Sigma_{p}^{\varepsilon} \backslash \hat{K}, G(\mu) \leqq s$, we know that $\exp (-t v)(\mu)$ reaches the region $G \leqq-T+1$ for some $t \in[0, \widetilde{C}(s-(-T+1))]$. Hence $\mu \notin \Gamma_{+}$.

Similarly, we define the incoming tail $\Gamma_{-}$, which is a closed $v$-invariant set contained in the region $G<T-1$, such that $\Gamma_{-} \cap\{G \geqq s\}$ is compact for every $s<T-1$. Let $K=\Gamma_{+} \cap \Gamma_{-}$, which is then a compact $v$-invariant set, contained in the region $-T+1<G<T-1$.

Proposition A.2. If $\Gamma_{-} \neq \emptyset$ ( or if $\Gamma_{+} \neq \emptyset$ ), then $K \neq \emptyset$.

Proof. Let $\varrho \in \Gamma_{-}$. Then $\{\exp t v(\varrho) ; t \geqq 0\}$ is contained in a compact set $L$, so we have $\exp t_{j} v(\varrho) \rightarrow \varrho_{0} \in \Sigma_{p}^{\varepsilon}$, for some $\varrho_{0} \in \Sigma_{p}^{\varepsilon}$ and some sequence $t_{j} \rightarrow \infty$. Put $\varrho_{j}=\exp t_{j} v(\varrho)$. For every $T>0$, we then have $\exp t v\left(\varrho_{j}\right) \rightarrow \exp t v\left(\varrho_{0}\right)$, uniformly for $t \leqq T$. But $\exp t v\left(\varrho_{j}\right)=\exp \left(t_{j}+t\right) v(\varrho) \in L$ for $j$ large enough, so $\exp t v\left(\varrho_{0}\right) \in L$, for $t \leqq T$. Here $T$ may be as large as we like, so $\varrho_{0} \in K$.

We next define the true tails $\mathscr{T}_{ \pm}=\Gamma_{ \pm} \backslash K$. On $\Sigma_{p}^{\varepsilon}$, we have the symplectic volume, which is $H_{p}$-invariant. For any $s<T-1$, the set $\mathscr{T}_{-} \cap\{G \geqq s\}$ is bounded and hence of finite volume. On the other hand, $\exp t H_{p}\left(\mathscr{T}_{-} \cap\{G \geqq s\}\right) \searrow \emptyset$, when $t \rightarrow+\infty$, so its volume tends to zero when $t \rightarrow+\infty$. On the other hand, this volume is constant, so it has to be zero for all $t$, and hence $\operatorname{Vol}\left(\mathscr{T}_{-} \cap\{G \geqq s\}\right)=0$, for all $s$. Hence $\operatorname{Vol}\left(\mathscr{T}_{-}\right)=0$. The same argument works for $\mathscr{T}_{+}$, and we have proved

Proposition A.3. $\operatorname{Vol}\left(\mathscr{T}_{ \pm}\right)=0$.

We also have

Proposition A.4. The following statements are equivalent:

(a) $\mathscr{T}_{+} \neq \emptyset$.

(b) $\mathscr{T}_{-} \neq \emptyset$.

(c) If we put $K_{\alpha}=\left\{\varrho \in \Sigma_{p}^{\varepsilon}\right.$; $\left.\operatorname{dist}(\varrho, K) \leqq \alpha\right\}$, then $K$ and $K_{\alpha} \backslash K$ are non-empty for every $\alpha>0$. 
Proof. We have already proved that if $\mathscr{T}_{+}$or $\mathscr{T}_{-}$is non-empty, then $K$ is nonempty, and the proof also shows that $K_{\alpha} \backslash K$ is non-empty for every $\alpha>0$. It remains to prove that (c) $\Rightarrow(\mathrm{a})$, and that $(\mathrm{c}) \Rightarrow(\mathrm{b})$.

Assume (c). The volume of $K_{\alpha} \backslash K$ is non-zero for every $\alpha>0$, so $K_{\alpha} \backslash K$ must contain points which are not in $\mathscr{T}_{-}$, and hence not in $\Gamma_{-}$. Take a sequence of such points; $\varrho_{j} \in K_{\alpha} \backslash\left(K \cup \Gamma_{-}\right)$which converges to $\varrho_{0} \in K$. The corresponding trajectories $\gamma_{j}:\left[0,+\infty\left[\ni t \rightarrow \exp t v\left(\varrho_{j}\right)\right.\right.$, then go to infinity as $t \rightarrow+\infty$, and we let $\varrho_{j}^{T}$ be the point of intersection with the hypersurface $G=T$. Then $\varrho_{j}^{T}=\gamma_{j}\left(t_{j}\right)$, where $t_{j} \rightarrow \infty, j \rightarrow \infty$. After passing to a subsequence, we can assume that $\varrho_{j}^{T} \rightarrow \varrho_{0}^{T}$. It is easy to see that $\varrho_{0}^{T} \in \mathscr{T}_{+}$, and we have proved that (c) implies (a). The proof of the other implication is, of course, the same.

Remark A.5. We could restrict the preceding discussion to $\Sigma_{p}=p^{-1}(0)$. Then $\Gamma_{ \pm}^{0}, K^{0}, \mathscr{T}_{ \pm}^{0}$ are defined as before, and Propositions A.1 and A.2 remain valid as well as the implications (a) $\Rightarrow$ (c) and (b) $\Rightarrow$ (c) in Proposition A.4. For the opposite implication $(\mathrm{c}) \Rightarrow(\mathrm{a})$, we need to approach $K^{0}$ by a sequence $\varrho_{j}$ which is not in $\mathscr{T}_{-}^{0}$. Everything goes through as before, if we assume that $d p \neq 0$ everywhere on $\Sigma_{p}$. In fact, we then have the $H_{p}$-invariant positive smooth Liouville form $\omega / d p$ on $\Sigma_{p}$, where $\omega$ denotes the symplectic volume form.

Hopefully, the above discussion could be a first step towards general refined choices of escape functions. In this paper, we only need a rather rough and intuitively obvious result about such choices.

Increasing $\widetilde{K}$ if necessary, we may assume that $K \subset \widetilde{K}$. Let $H_{T}=\left\{\varrho \in \Sigma_{p}^{\varepsilon}\right.$; $G(\varrho)=T\}$, and notice that we have the diffeomorphism, $\kappa_{+}: \mathbf{R} \times H_{T} \rightarrow \Sigma_{p}^{\varepsilon} \backslash \Gamma_{-}$, given by $\kappa_{+}(t, \varrho)=\exp t v(\varrho)$. Similarly, we have a diffeomorphism $\kappa_{-}: \mathbf{R} \times H_{-T} \rightarrow \Sigma_{p}^{\varepsilon} \backslash \Gamma_{+}$. Let $0<f_{+} \in C^{\infty}\left(\Sigma_{p}^{\varepsilon} \backslash \Gamma_{-}\right)$be $\geqq v(G)$ with equality in $\{G \geqq T\}$ and outside a compact set in $\{-T \leqq G \leqq T\}$. Let $G_{+} \in C^{\infty}\left(\Sigma_{p}^{\varepsilon} \backslash \Gamma_{-}\right)$be the solution of $v\left(G_{+}\right)=f_{+}$, with $G_{+}=T$ on $H_{T}$. Then $G_{+}=G$ in $\{G \geqq T\}$, and outside a compact set in $\{-T \leqq G \leqq T\}$. We also have $G_{+} \leqq G$, and by choosing $f_{+}$large enough, we may arrange so that

$$
\varlimsup_{\varrho \rightarrow \Gamma_{-} \cup H_{-T}} G_{+} \leqq-T .
$$

Let $f_{-}, G_{-}$have the completely analogous properties.

Let $\chi_{+}, \chi_{-} \in C^{\infty}(\mathbf{R} ; \mathbf{R})$ satisfy $\chi_{+}+\chi_{-}=t$ and have the properties given by the picture:

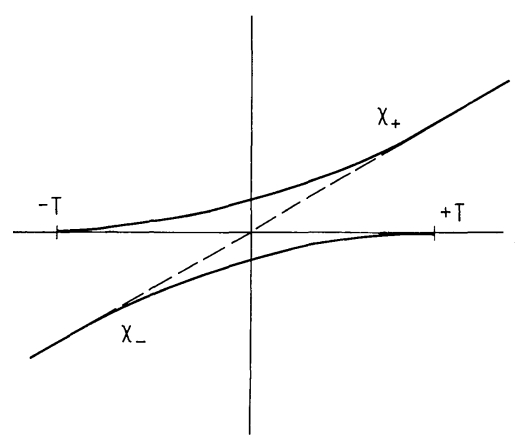

Fig. 1 
Put $\tilde{G}=\chi_{+} \circ G_{+}+\chi_{-} \circ G_{-}$. Here $\chi_{+} \circ G_{+}$can be smoothly extended by 0 in $\Gamma_{-}$, while $\chi_{-} \circ G_{-}$can be similarly extended to $\Gamma_{+}$. Thus $\tilde{G} \in C^{\infty}\left(\Sigma_{p}^{\varepsilon}\right)$. By the construction, we have $\widetilde{G}=G$ in $\{G \geqq T\} \cup\{G \leqq-T\}$, and outside a compact set in $\{-T \leqq G \leqq T\}$.

We have

$$
v(\widetilde{G})=\left(\chi_{+}^{\prime} \circ G_{+}\right) f_{+}+\left(\chi_{-}^{\prime} \circ G_{-}\right) f_{-} \geqq 0,
$$

while $\nabla G=\left(\chi_{+}^{\prime} \circ G_{+}\right) \nabla G_{+}+\left(\chi_{-}^{\prime} \circ G_{-}\right) \nabla G_{-}$. We conclude:

Proposition A.6. Given $G, T$ as above, we can find a new escape function $\widetilde{G}$, equal to $G$ outside a compact set, such that on $\Sigma_{p}^{\varepsilon}$ we have $\widetilde{G}=0$ in a neighborhood of $K=\Gamma_{+} \cap \Gamma_{-}$and such that locally uniformly on $\Sigma_{p}^{\varepsilon}$ :

$$
v(\tilde{G}) \geqq C_{0}^{-1}|\nabla \tilde{G}| .
$$

\section{References}

(Further references about resonances can be found for instance in [6]. Here we limit ourselves to the works that play a role for the special problems and techniques under consideration.)

1. Abraham, R., Marsden, J.: Foundations of mechanics. New York: Benjamin/Cummings 1978

2. Boutet de Monvel, L., Grigis, A., Helffer, B.: Parametrixes d'opérateurs pseudodifferentiels à caractéristiques multiples. Astérisque 34-35, 93-121 (1976)

3. Cordoba, A., Fefferman, C.: Wavepackets and Fourier integral operators. Commun. Partial Differ. Equations 3, 979-1005 (1978)

4. Gérard, C.: Asymptotique des pôles de la matrice de scattering pour deux obstacles strictement convexes (preprint)

5. Guillope, L.: Private communication

6. Helffer, B., Sjöstrand, J.: Résonances en limite semi-classique. Mémoire de la S.M.F., $n^{\circ}$ 24/25, Suppl. an Bull. de la S.M.F., 114 (1986), fasc. 3

7. Ikawa, M.: On the poles of the scattering matrix for two convex obstacles. J. Math. Kyoto Univ. 23, 127-194 (1983). An addendum in J. Math. Kyoto Univ. 23, 795-802 (1983)

8. Ikawa, M.: Precise information on the poles of the scattering matrix for two strictly convex obstacles. Preprint, also in the proceedings of the Journées des E.D.P. at St. Jean de Monts, 1985

9. Melin, A., Sjöstrand, J.: Fourier integral operators with complex valued phase functions. Lecture Notes in Mathematics, Vol. 459. Berlin, Heidelberg, New York: Springer, pp. 120-223

10. Reed, M., Simon, B.: Methods of modern mathematical physics, I-IV. New York: Academic Press, pp. 1974-82

11. Sjöstrand, J.: Analytic wavefront sets and operators with multiple characteristics. Hokkaido Math. J. 12, 392-433 (1983)

12. Sjöstrand, J.: Parametrices for pseudodifferential operators with multiple characteristics. Ark. Math. 12, 85-120 (1974)

13. Sjöstrand, J.: Singularités analytiques microlocales. Astérisque 95 (1982)

14. Sjöstrand, J.: Semiclassical resonances generated by non-degenerated critical points. Preprint of the University of Lund (1986)

15. Ralston, J.V.: On the construction of quasimodes associated with stable periodic orbits. Commun. Math. Phys. 51, 219-242 (1976)

16. Colin de Verdière, Y.: Quasimodes sur les variétés riemanniennes. Invent. Math. 43, 15-52 (1977)

17. Candelpergher, B., Nosmas, J.C.: Quantification et approximations semiclassiques. Colloque de Saint Jean de Monts (1982) 
18. Voros, A.: The W.K.B. method for non-separable systems. Actes du congrès international de géométrie symplectique et physique mathématique, Aix (1974)

19. Duistermaat, J.J.: Oscillatory integrals, lagrangian immersions and unfolding of singularities. Commun. Pure Appl. Math. 27, 207-281 (1974)

20. Pollak, E.: A quasiclassical model for resonance widths in quantal colinear reactive scattering. J. Chem. Phys. 76, 5843-5848 (1982)

21. Gutzwiller, M.C.: Periodic orbits and classical quantization conditions. J. Math. Phys. 12, 343-358 (1971)

Communicated by B. Simon

Received April 29, 1986; in revised form September 8, 1986 
